\title{
Experimental Investigation into the Turbulence Flow Field of In-Flight Round Jets
}

\author{
Anderson R. Proença*, Jack L. T. Lawrence ${ }^{\dagger}$ and Rod H. Self ${ }^{\dagger}$ \\ University of Southampton, $U K$
}

\begin{abstract}
In this paper, insight is provided into the modelling of single-point and two-point statistics of a subsonic round jet discharged into a moving ambient medium. An experimental campaign has been performed comprising two round, unheated air flows - a central 'jet' at Mach number equal to 0.6 surrounded by a slower 'flight' flow. Constant temperature hot-wire anemometry was used to measure both the axial and radial velocity fluctuations within the turbulent jet flow field. A Mach 0.6 jet was discharged into the flight flow, which ranged from zero up to Mach 0.3. The data show that the degree to which the jet stretches with increasing flight velocity can be discerned with knowledge of the decay of the mean velocity field downstream of the end of the jet's potential core. This stretching factor can then be used to predict the changes in the static jet turbulence statistics for the in-flight case. Additionally, in the region of high turbulence kinetic energy, the two-point statistics can be estimated using information about the single-point statistics and the local mean velocity. Empirical models for the in-flight jet's shear stresses, cross-correlations, and power spectral density functions are presented and compared to those derived for the static jet case.
\end{abstract}

\section{Nomenclature}

$C_{\delta} \quad=\quad$ ratio between the shear layer width and the jet nozzle exit diameter

$C_{\mathrm{f}} \quad=\quad$ ratio between the jet exit velocity and the jet-to-flight relative velocity

$D \quad=$ nozzle exit diameter $[\mathrm{m}]$

$M \quad=\quad$ jet Mach number at the nozzle exit

$R(\zeta, \tau)=$ space-time cross-correlation coefficient

$S(\zeta, \omega)=$ cross-power spectral density

St $\quad=$ Strouhal number

$T=$ Total temperature $\left[{ }^{\circ} \mathrm{C}\right]$

TI $=$ axial turbulence intensity

\footnotetext{
*Research Fellow, ISVR, University of Southampton, AIAA Member.

${ }^{\dagger}$ Research Fellow, ISVR, University of Southampton, AIAA Member.

$\$$ Professor, ISVR, University of Southampton, AIAA Member.
} 


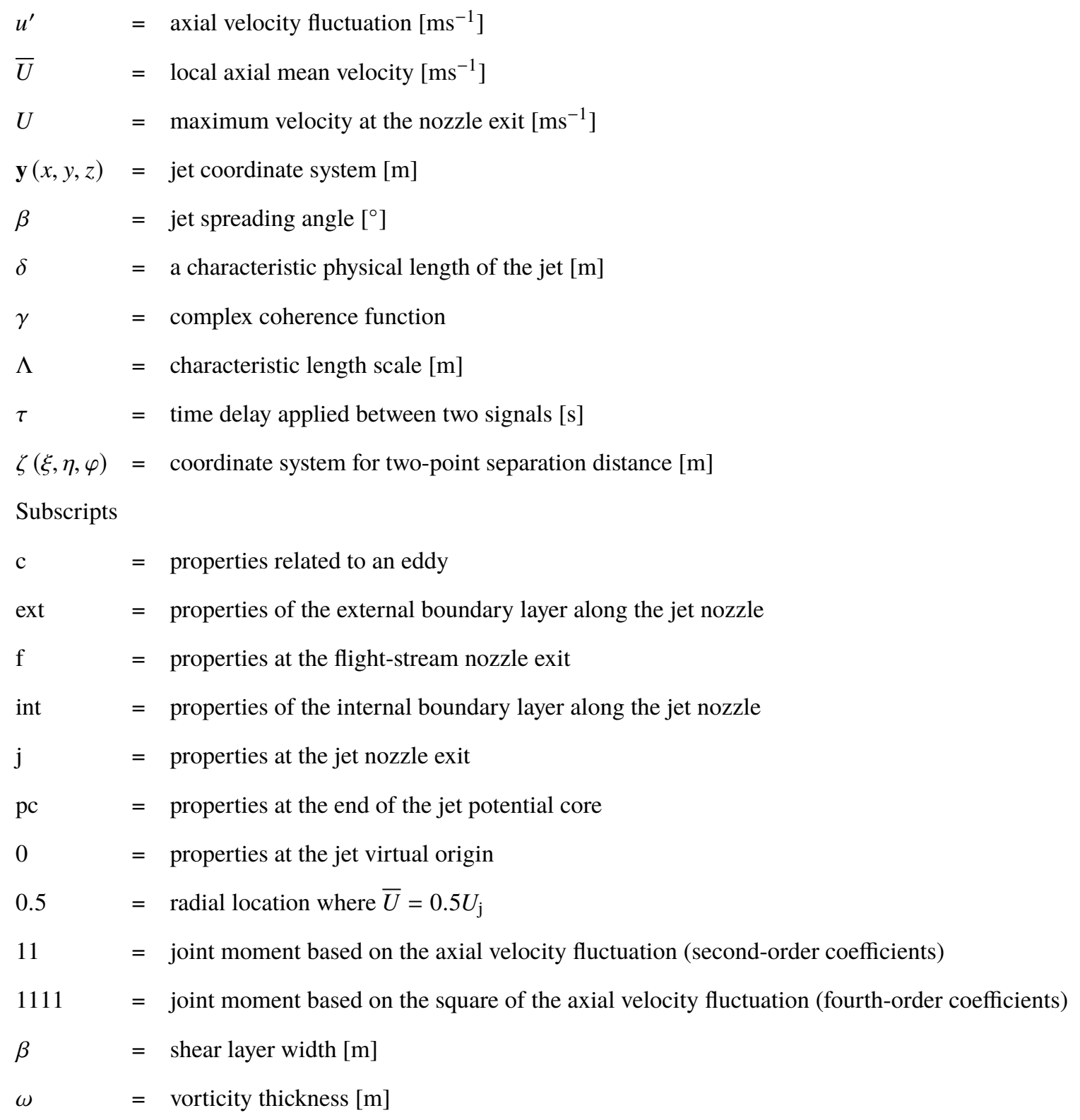

\section{Introduction}

HE jet exhausted from turbofan engines remains a dominant noise source of modern commercial aircraft during take-off. While jet mixing noise has been reduced to the point of being of secondary importance at other certification reference points, it still plays a major role at the sideline certification position. At this observer location, jet noise contributes between one-third and half of the total acoustic energy generated during take-off [1]. Additionally, the increasing interaction between the jet's hydrodynamic field and solid surfaces close by (e.g. the wing and high-lift devices) is set to lead to an increase in the low frequency region of the aircraft noise spectrum. During take-off, it is well-known that the presence of the ambient 'flight' airflow serves to stretch the jet exhausted from the engine. As well 
as reducing the magnitude of jet mixing noise, this stretching should, in theory, also lead to a reduction in jet installation noise compared to the static case. This is due both to the reduction in the magnitude of the jet's hydrodynamic shear layer instability and the fact that the hydrodynamic field is effectively moved away from the wing surface. However, forward flight effects on the jet flow and pressure fields are complex and have not been fully understood [2-[6]. The development of accurate models to predict in-flight installed jet noise is still an open problem.

In-flight jet mixing noise effects, measured in the acoustic far field, have been investigated extensively both in open jet wind tunnels [7.-9] and full-scale turbojet engines [10]. Scarce amounts of experimental data, however, exist regarding the unsteady flow field of in-flight jets. While several papers from the 1970s do describe measurements of single-point and two-point statistics of in-flight jets [11-14], their analyses are all limited with regards to the spectral content.

In this paper, the effects of forward flight on the turbulent flow field of a single-stream, isolated, subsonic, round jet are presented. The main aim is to demonstrate that the shear layer width collapses single-point and two-point statistics of in-flight jets in the initial and transitional regions (i.e. from the nozzle exit up to two jet potential core lengths). This survey is a direct extension of a recent study carried out for subsonic, static jets [15]. The turbulence flow field is measured using hot-wire anemometry. A subsonic $M_{\mathrm{j}}=0.6$ jet is discharged into both stationary and moving media. Single-point, single-component measurements are used to investigate the degree both to which the jet is stretched by the flight flow and to which the in-flight jet is self-similar. Power spectral density and autocorrelation functions are also studied to quantify the turbulence length-scales and time-scales. Measurements using two single-component probes and one two-component probe were both performed. Finally, an argument is made regarding the validity of using open jet wind tunnels to simulate in-flight jet noise. In most small-scale open-jet wind tunnels, the jet noise radiation propagates out through the wind tunnel shear layer before it is recorded. Thus, the study presented here also aims to show that the jet turbulence statistics of small-scale in-flight jets are not affected by the presence of the open jet wind tunnel shear layer. If so, any issues related to the far field measurement should then be linked with shear layer refraction effects, which are relatively well-understood [2, 16].

The paper is organised as follows. Section II introduces the experimental facility and methodology. In Section III, results for the mean axial velocity component along the jet centreline are analysed in order to define the virtual origin of the jet. The virtual jet origin parameter is then used to define a stretching factor to describe the global behaviour of the in-flight jet. Next, in Section III-C, the jet turbulence intensity is investigated and the dependency of the turbulence levels on the flight velocity at different jet locations is presented. In Section III-D, the analysis moves to the frequency domain where the strength of the velocity fluctuation along the jet is evaluated using the power spectral density. Finally, a discussion regarding the prediction of two-point statistics from single-point data is given before the main conclusions are presented. 


\section{Experimental Methodology}

\section{A. Experimental Facility}

The ISVR Doak Laboratory is an anechoic chamber, fully anechoic down to a frequency of $400 \mathrm{~Hz}$. The facility has dimensions approximately of $15 \mathrm{~m}$ long by $7 \mathrm{~m}$ wide by $5 \mathrm{~m}$ high. A 'core' air jet is supplied by a high-pressure compressor-reservoir system. Single stream jet measurements can be performed on flow regimes characteristic of civil aircraft and for $1 / 50^{\text {th }}$-scale experiments. The jet rig is capable of achieving a controlled exit acoustic Mach number range of between 0.15 and 1. Further information regarding the Doak Jet Rig can be found in previous papers and reports [15, 17, 18].

The Doak Lab has been recently upgraded to perform forward flight simulations. The co-annular 'flight' jet of the new Flight Jet Rig is supplied by a backward curved centrifugal fan. The flight jet exit Mach number ranges from 0.015 to 0.3 . The upper limit was designed to match full-scale aircraft speeds during take-off (i.e. approximately $100 \mathrm{~m} / \mathrm{s}$ ). The newly built facility is shown in Fig. 1
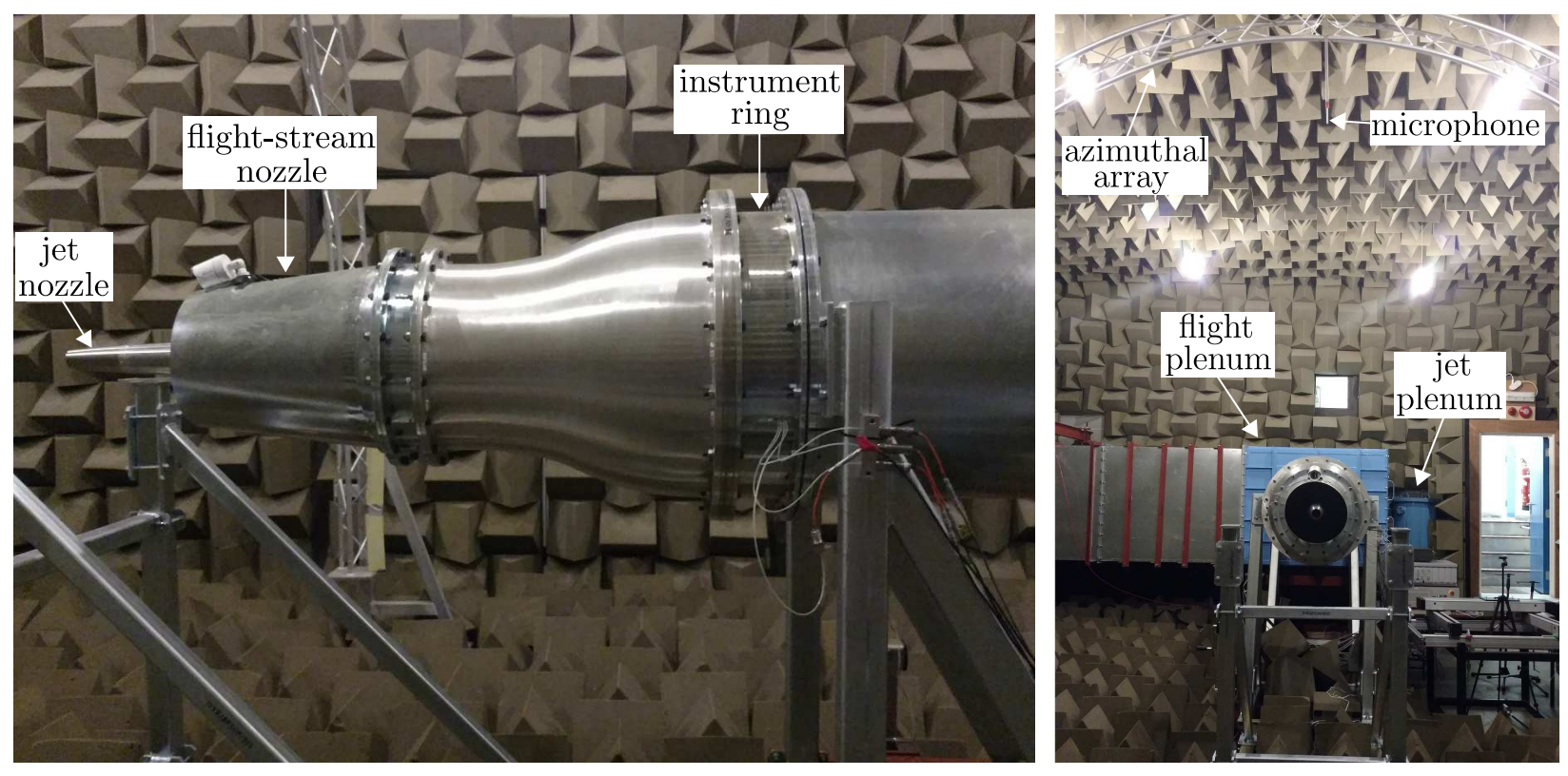

Fig. 1 The new Flight Jet Rig in the Doak Laboratory at the University of Southampton, UK

A conical core jet nozzle geometry with a low $3^{\circ}$ convergence half angle was designed to avoid separation of the flight-stream boundary layer that develops on the external surface of the core jet pipe. The conical flight jet nozzle has a $7^{\circ}$ convergent half angle. Figure 2 shows the geometry of the jet nozzle and flight-stream nozzle used in the Flight Jet Rig. Further information about the Flight Jet Rig can be found in [18].

In order to perform single-point and two-point hot-wire measurements of the core jet turbulence field, the laboratory was equipped with two ISEL traverse systems mounted adjacent to the Flight Jet Rig. The traverse systems allowed independent movement along the $\mathrm{x}, \mathrm{y}$ and $\mathrm{z}$ planes. The maximum stroke along each axis equated to 16 core jet nozzle 

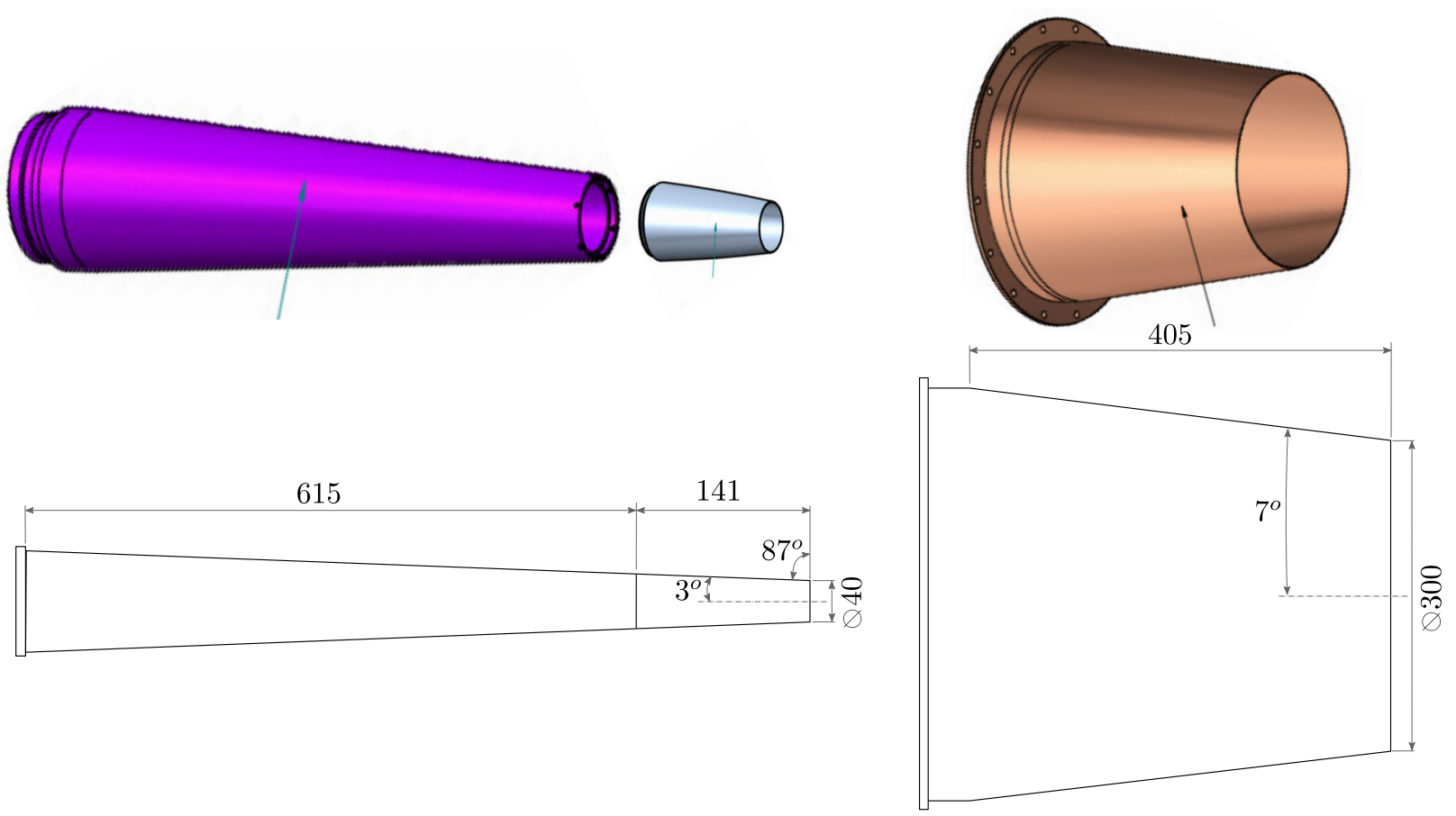

(a)

(b)

Fig. 2 (a) $40 \mathrm{~mm}$ diameter core jet nozzle geometry and (b) $300 \mathrm{~mm}$ diameter flight jet nozzle geometry. Dimensions in $\mathbf{m m}$

diameters. The origin is defined at the centre of the core jet nozzle exit, $x=y=z=0$.

\section{B. Instrumentation and data acquisition}

The core and the flight jet exit aerodynamic Mach numbers were defined using the pressure ratio between the total upstream pressure and the pressure at the nozzle exit together with the isentropic flow equations. It was assumed that the static pressure at the nozzle exit was equal to the ambient chamber pressure. The total pressures of the core and flight jets were measured upstream of the nozzle exit, in the instrument ring (see Fig. 1). General purpose type K thermocouple probes mounted upstream of the jet and flight nozzles were used to calculate the speed of sound of the core and flight jets.

The core and flight jet unsteady velocity was measured using a constant temperature anemometry (CTA) hot-wire system. A StreamLine Pro CTA system produced by Dantec Dynamics was used. This system was built with two CTA modules, allowing simultaneous measurement of either two single-component hot-wire probes or a cross-wire probe (axial and lateral velocity components). The hardware setup was performed within the environment of the StreamLine Pro software.

Single, normally-oriented hot-wires and cross-wire probes f $^{\text {were }}$ used in the experiments. The hot-wire probes were calibrated using a dedicated, automatic Dantec calibrator. The calibration was performed before and after the tests

\footnotetext{
*Dantec 55P11 and 55P61 miniature probes, both configurations are made of 5 $\mu \mathrm{m}$ diameter, $1.25 \mathrm{~mm}$ long plated tungsten wires.
} 
were conducted. The uncertainty due to the calibrator unit and the linearisation of the calibration data is estimated by the manufacturer to be within $\pm 0.5 \%$ of the nominal velocity [19]. Ambient temperature variation between tests was relatively small (e.g. $\left.\pm 2^{\circ} \mathrm{C}\right)$ Thus, the CTA overheat was adjusted before the tests and a temperature probe was used to measure the flow temperature fluctuations during the tests. The hot-wire raw voltage data was then corrected to account for the temperature fluctuation as suggested by the CTA manufacturer. Errors due to the drift in flow and ambient properties are expected not to exceed $1 \%$ of the sample velocity [19]. More information about the calibration procedure, other sources of error and experimental uncertainty can be found in Proença et al. [18, 20].

The hot-wire probe was traversed radially across the two jets at axial stations from the nozzle exit and up to 12 core jet diameters downstream. Additionally, two-point measurements were carried out using two single-wire probes. The reference probe was fixed at a given axial location and the moving probe was positioned at 20 different axial locations downstream of the reference probe. The reference probe was always located along the lipline, at $x / D_{\mathrm{j}}=2,4,6$, and 8 . In all tests, the core jet velocity was kept constant at $M_{\mathrm{j}}=0.6$. The flight Mach number was varied from 0 (i.e. the static case) to 0.3 , in 0.05 increments. All data were acquired using a 24-bit National Instruments dynamic signal acquisition system. The steady ambient chamber and mean rig flow data were sampled at $1 \mathrm{kHz}$. The unsteady hot-wire measurements were each recorded with a sampling rate of $100 \mathrm{kHz}$ for ten seconds.

Before the results are discussed, definitions about the jet properties and the statistical joint moments are introduced.

\section{Jet properties}

The shear layer width $\left(\delta_{\beta}\right)$, spreading rate $\left(\beta_{1}\right.$ and $\left.\beta_{2}\right)$ and the three regions of the jet are defined in Fig. 3(a). Recently hot-wire measurements on a static jet suggest that the spreading rate is constant at least up to two potential core lengths. Therefore, the shear layer width grows more rapidly in the initial region in comparison to the transitional region [15]. The jet spreading growth rate in the initial region is function of the decrease in the potential core radius and, therefore, the total potential core length. These parameters depend strongly on the flight velocity and empirical relationships are demonstrated in the Results section.

The spreading rate $\beta_{1}$ and, consequently, the shear layer width, are found either by direct measurements of the jet mean velocity profiles or by the definition of a virtual origin located upstream of the jet nozzle exit. The virtual origin, $X_{0}$, is more commonly used to define the spreading rate in the jet fully-developed region $\left(\beta_{2}\right)$. Many diameters downstream (i.e. $x>20 D_{\mathrm{j}}$ ), the inverse of the axial mean velocity decays linearly. When the velocity decay is plotted as $U_{\mathrm{j}} / U$, the intercept of a best fit of the experimental data with the abscissa defines the virtual origin [21, 22]. The same procedure is applied in this work, but the mean velocity decay in the two first regions of the jet, instead of the fully-developed region, is used. Figure 3(b) illustrates the inverse of the mean velocity profile and the location of the

\footnotetext{
${ }^{\dagger}$ The hot-wire mappings and two-point tests were carried out within a week.
} 
virtual origin. Experimental data of a static $M_{\mathrm{j}}=0.6$ jet was used. The linear fit was obtained using only the points where $U_{\mathrm{j}} / U>1.05$. Figure $3(\mathrm{c})$ shows the location of the virtual origin in comparison to the jet nozzle. The virtual origin is used to define the spreading rate from the line with origin at $X_{0}$ and passing by the nozzle lip at $x / D_{\mathrm{j}}=0$.

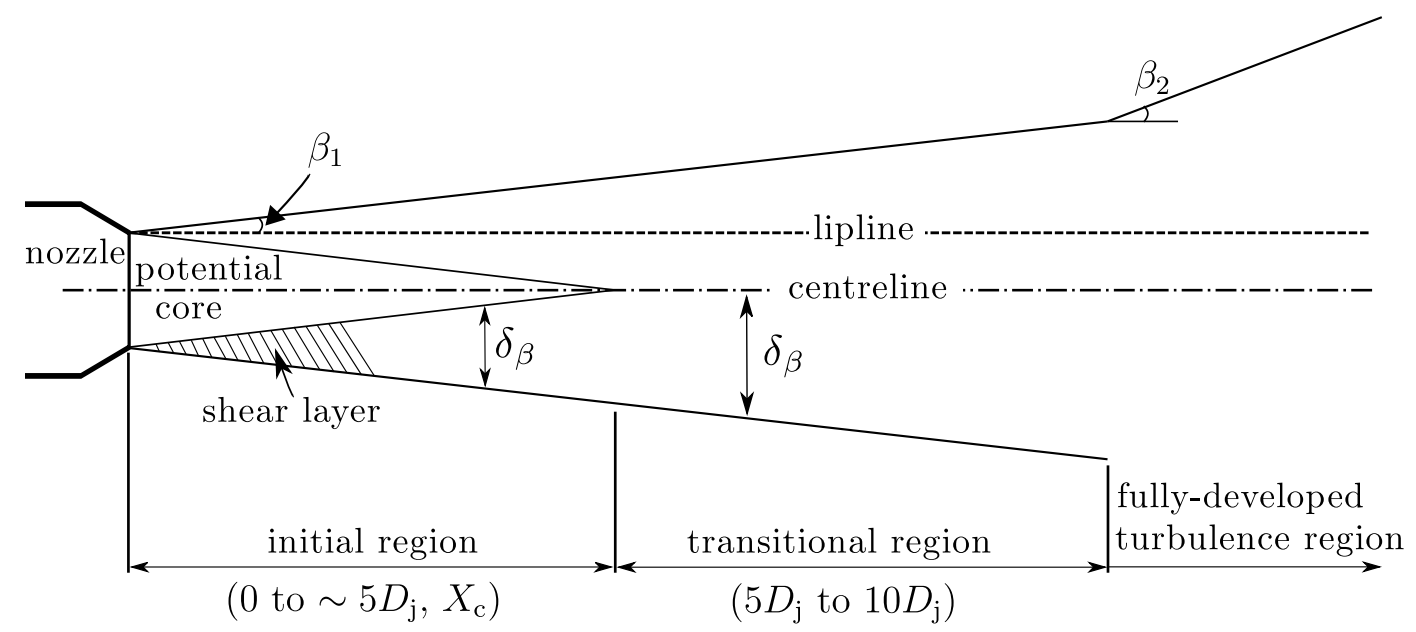

(a)

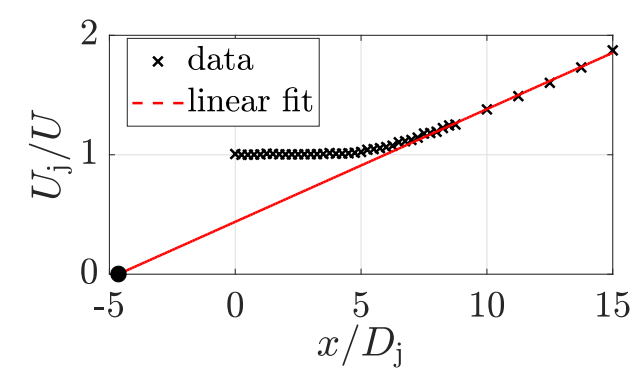

(b)

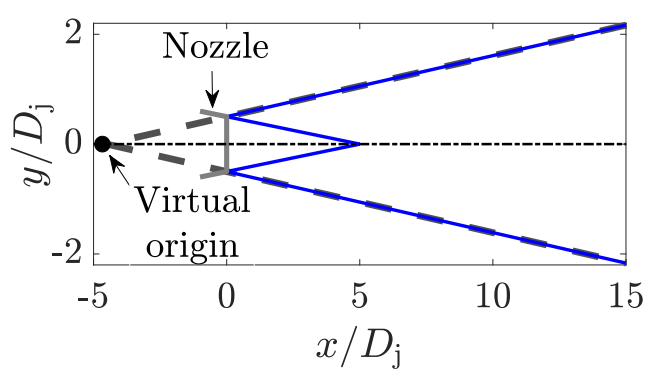

(c)

Fig. 3 (a) Definition of the jet properties and jet regions used in the text. (b) Variation of the inverse of the mean velocity along the jet centreline with axial distance. (c) Schematic showing the location of the jet virtual origin with respect to the jet nozzle.

\section{Data post-processing}

The hot-wire voltage data was corrected for temperature fluctuations and then converted to velocity using fourth-order polynomial coefficients obtained from the calibration procedure. The instantaneous velocity and velocity fluctuation samples of all test points were stored in matrices using the software Matlab. Central moments (mean value, variance, skewness and kurtosis) were recorded.

The second-order space-time cross-correlation coefficients discussed in the text are defined by, 


$$
R_{11}(x, \xi, \tau)=\frac{\overline{u^{\prime}(x, t) u^{\prime}(x+\xi, t+\tau)}}{\sqrt{\overline{u^{\prime 2}}(x) \overline{u^{\prime 2}}(x+\xi)}}
$$

whilst the fourth-order cross-correlation coefficients used in this work are defined by,

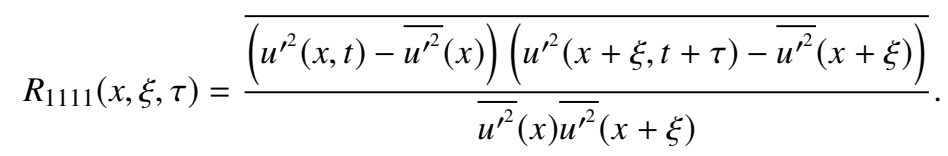

In the frequency domain analysis, the power spectral density (PSD) was calculated at each point in the jet using Welch's method. The velocity signal was split into 100 segments each with 50\% overlap. The fast Fourier transform of each segment was taken using a Hanning window. Then an average over the estimates of each segment was taken. A similar procedure was used to calculate the cross-power spetral density (CPSD). The PSD and the CPSD were used to calculate the coherence by the following equation,

$$
\gamma_{11}(x, \xi, \omega)=\frac{\operatorname{CPSD}(x, \xi, \omega)}{\sqrt{\operatorname{PSD}(x, \omega) \operatorname{PSD}(x+\xi, \omega)}} .
$$

Finally, the characteristic length scales were defined as the location in which the cross-correlation coefficients and the magnitude of the coherence are equal to 1/e. This assumes that the joint moments can be modelled as a one-term exponential function and, therefore, the integration of the area under the coefficients is 1/e. This assumption is accurate in jet regions where the turbulence intensity is high [15, 23, 24].

\section{Results}

In this section, the analysis of the experimental data is presented. Firstly, the elongation of the jet potential core and the variation of the velocity decay rate along the centreline with flight velocity are analysed. These two parameters are then used to define a virtual origin of the two initial regions of the jet (i.e. $2 X_{\mathrm{c}}$ ). The jet spreading rate, $\beta_{1}$, and shear layer width, $\delta_{\beta}$ are calculated and compared to experimental data. Finally, the parameter $\delta_{\beta}$ is used in the subsequent sections to scale the turbulence intensity, power spectral density, cross-correlation and coherence of several in-flight jet locations and jet-to-flight velocity difference.

\section{A. Centreline Mean Velocity and Jet Virtual Origin}

The mean axial velocity profile along the core jet centreline is used to obtain the flight effects on the potential core length $X_{\mathrm{c}}$ and the velocity decay downstream of the end of the potential core. Results of the mean axial velocity field along the centreline of the $M_{\mathrm{j}}=0.6$ jet at one static and three flight speeds are shown in Fig. 4(a) The axial location is normalised by the jet nozzle diameter and the local mean velocity is normalised by the maximum mean velocity on the core jet centreline. The potential core length is defined as the distance from the nozzle exit to the location at which the 
mean velocity profile decays to $0.98 U_{\mathrm{j}}$.

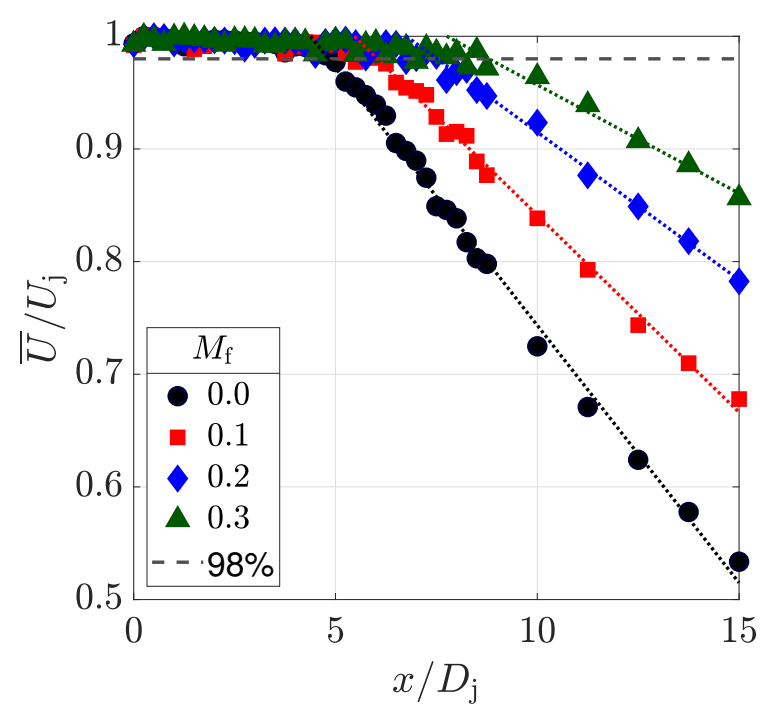

(a)

Fig. 4 Normalised mean axial velocity data along the centreline of an $M_{j}=0.6$ jet discharged into stationary and moving media.

Downstream of the end of the potential core, the gradient of the velocity decay is seen to decrease linearly with increasing flight velocity. As expected, the potential core length increases considerably with flight velocity. The decay of the mean axial velocity can be defined as the inverse of the axial distance or by Witze's semi-empirical equation for static jets [25]. Both approximations are also seen to work well in flight condition. This is true at least for axial locations less than twice the potential core length.

The variation of the jet potential core length with flight velocity is presented in Fig. 5. Experimental data is displayed in Fig. 5(a) by black circles. Linear and exponential fits are calculated and indicated by solid and dashed lines, respectively. The 95\% confidence intervals are also shown. In Fig. 5(b), the two best fit models to the experimental data are plotted against results from the literature [11, 26].

The differences seen between the empirical models shown in Fig. 5(b) were expected. Determining the length of the potential core requires full knowledge of the jet's boundary conditions. Witze used a proportionality constant function of the jet exit Mach number to approximate the length of the potential core. According to his results, the jet potential core varies approximately from $4.5 D_{\mathrm{j}}$ to $5 D_{\mathrm{j}}$ for jets at $M_{\mathrm{j}}=0.2$ and $M_{\mathrm{j}}=0.8$, respectively. Additionally, nozzles with different contraction ratio will produce different boundary layer properties at the nozzle exit, leading to different jet spreading and potential core lengths [18]. Thus, the difference in the boundary conditions of the experiments described here and by Morris [11] is a strong hypothesis to explain the relatively consistent offset seen in the variation of the potential core length with flight velocity of these two datasets. 


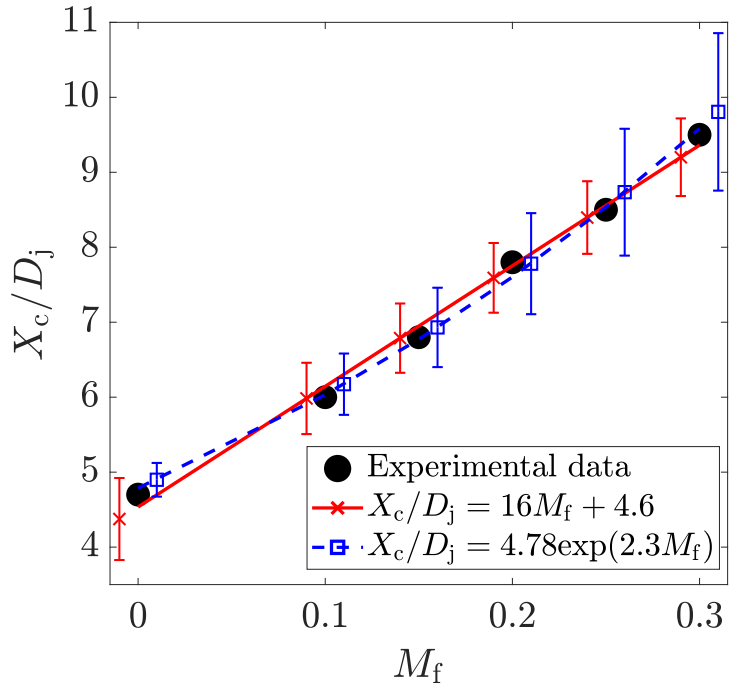

(a)

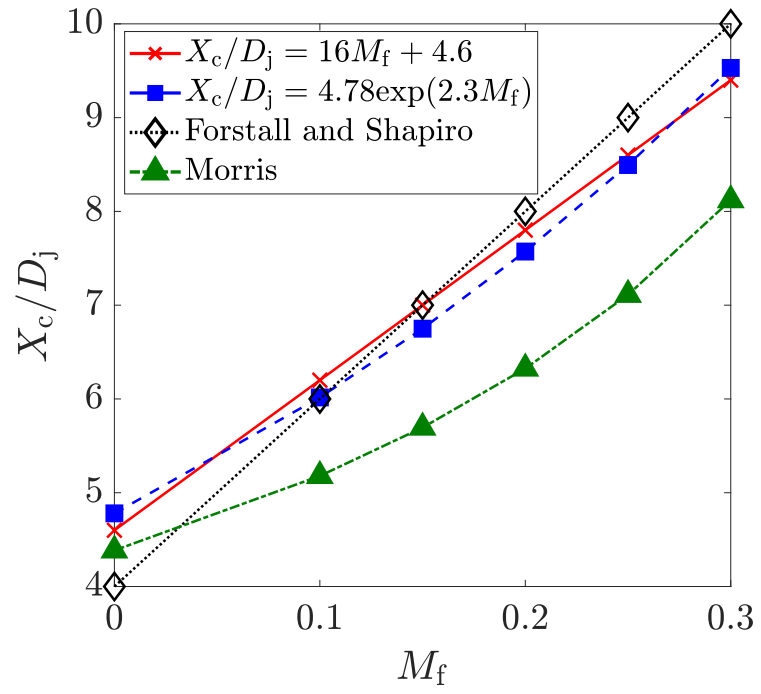

(b)

Fig. 5 (a) Experimental data and best-fit trend for the core jet potential core length as a function of flight Mach number. (b) Comparison between the best-fits shown in Fig. 5(a)] and empirical models from Forstall and Shapiro [26] and Morris [11]

As the confidence intervals shown in Fig. 4 slightly favour the linear fit, the jet potential core length as function of the flight Mach number used herein is defined as,

$$
\frac{X_{\mathrm{c}}}{D}=16 M_{\mathrm{f}}+4.6 .
$$

The analysis then follows to obtaining the virtual origin defined in Sec. II.C. The interest in the virtual origin, here, is to evaluate whether it provides a robust approximation of the spreading rate of an in-flight jet and, therefore, whether it can be used to predict both the correct length scales used in jet noise source models and the correct strength of the scattered hydrodynamic field for installed jet cases. As the potential core length, $X_{\mathrm{c}}$, is seen to vary linearly with flight velocity, this length is used to normalise the axial distance rather than the jet diameter and to define the virtual jet origin, $X_{0}$, as shown in Fig. 6 for four flight velocities. The same agreement was obtained for several other intermediate flight velocities studied.

The data leads to the following empirical relationship between the virtual origin and flight velocity,

$$
\frac{X_{0}}{D_{\mathrm{j}}}=-\left(18.4 M_{\mathrm{f}}+5.3\right) .
$$

A simple relationship can also be expressed between the virtual origin and the jet potential core length, as $X_{0}=-1.15 X_{\mathrm{c}}$. The minus sign indicates that the origin is defined upstream of the core jet nozzle exit. Eq.5 is valid for both static and in-flight jets, provided the flight Mach number is lower than or equal to 0.3 (i.e. for incompressible flight velocities). 


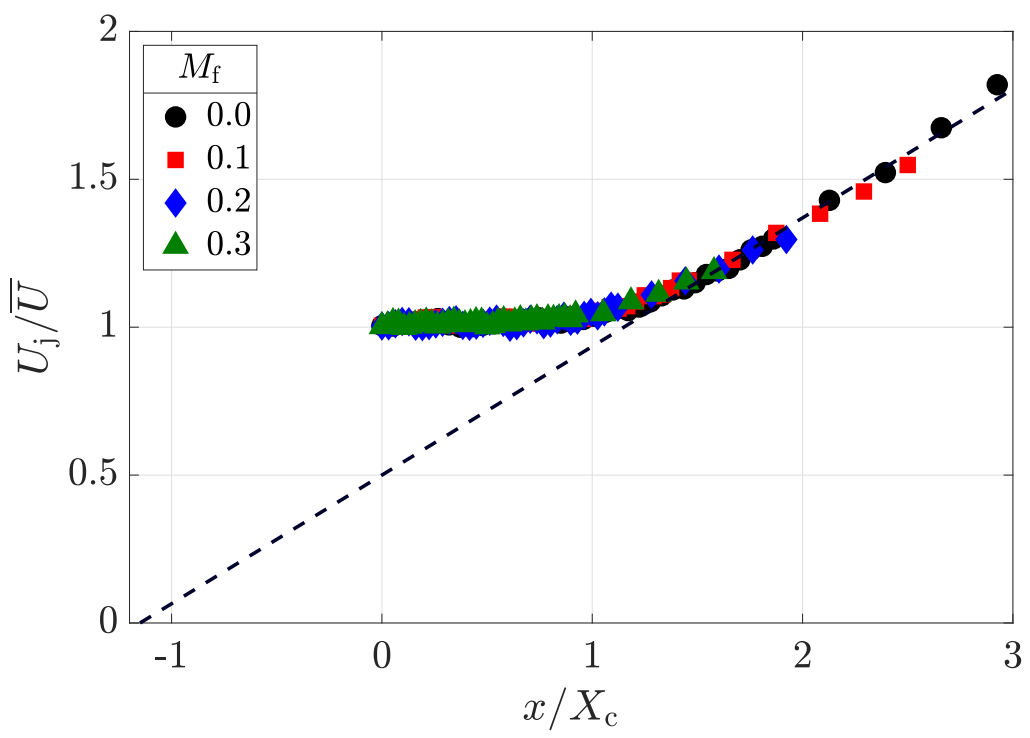

Fig. 6 Inverse velocity profile and estimation of the virtual origin of the core jet at various flight jet velocities

Further investigation is necessary to confirm the behaviour at higher compressible flight velocities, however, this is beyond the scope of the current work.

\section{B. Stretching Factor and Self-Similarity}

In this section, the jet spreading rate calculated using the virtual origin, defined by Eq. 5 , is compared to the jet spreading based on radial mean velocity profiles. In so doing, the self-similarity property of an in-flight jet is discussed. The stretching factor for an in-flight jet is important for two reasons. Firstly, the stretching of the jet affects the development of the characteristic turbulence length-scales. Secondly, the distance between the edge of the shear layer and a wing or flap surface is important in determining the strength of the hydrodynamic pressure field of a jet when it is installed on an airframe.

The spreading rate of the jet is defined using the virtual origin as follows,

$$
\beta=\arctan \left(\frac{0.5 D_{\mathrm{j}}}{X_{0}}\right)
$$

Both the spreading rate and stretching factor were determined experimentally by single-point mean axial velocity mappings. The resultant static and in-flight mean velocity field for the Mach 0.6 jet are shown in Fig.7 A selection of the mean velocity profiles measured in the experiments is represented by arrows. The colour scale is obtained via linear interpolation of the measured data. The spreading rates predicted using Eq.6 are indicated in the title of each graph and are represented as dashed lines.

It can be seen, in Fig. 77 that the spreading rate calculated from the jet virtual origin well captures the stretching of 

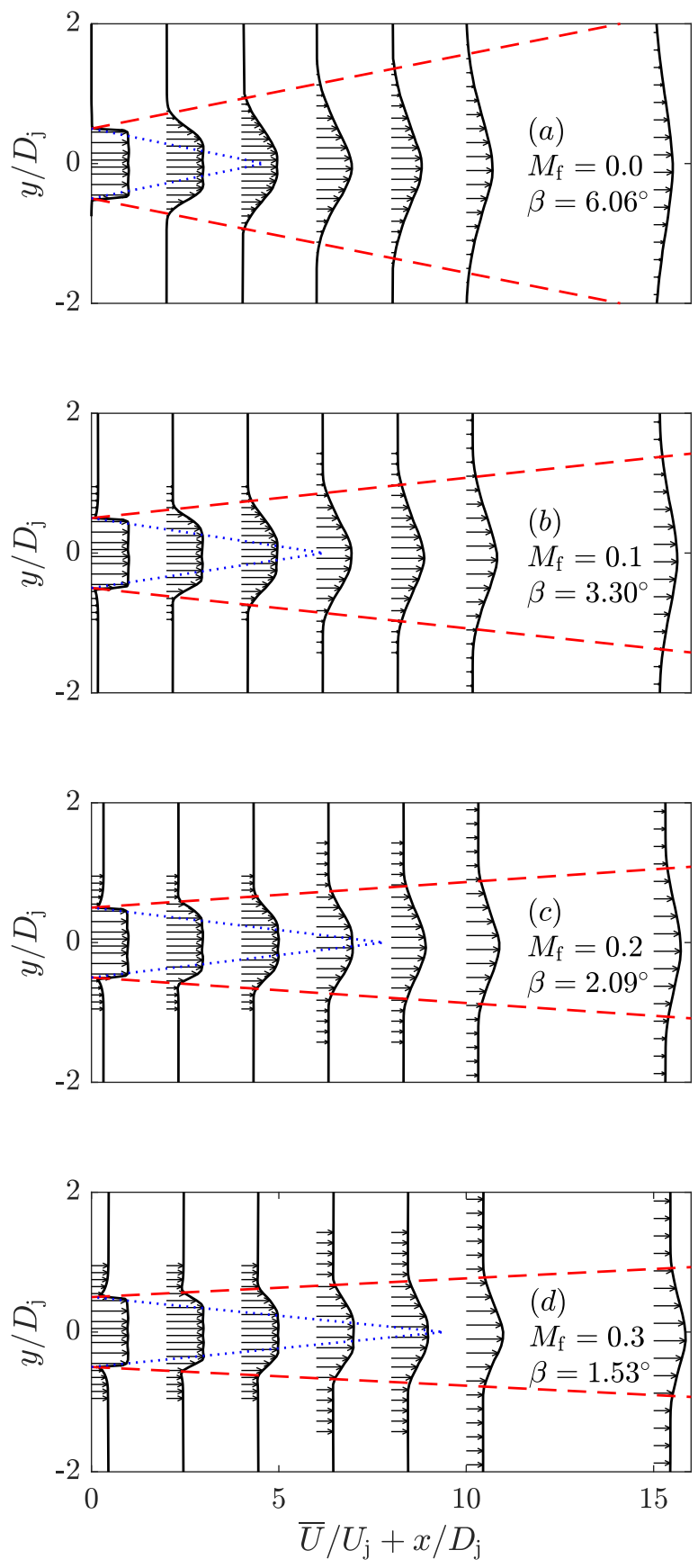

Fig. 7 Mean axial velocity distribution showing the change in the spreading rate of the jet with flight velocity as predicted using Eq. 7. The jet potential core lenght calculated using Eq. 4 and the jet spreading rate from Eq. 7 are indicated by the blue dotted lines and the red dashed lines, respectively 
the jet with increasing flight velocity. Note that, in figures 7 b) to (d), a low velocity region is seen close to the jet nozzle exit. This is due to the boundary layer that develops on the external surface of the jet nozzle. This external boundary layer effect on the jet flow velocity is negligible for axial locations greater than two core jet diameters downstream of the core nozzle.

Combining equations 4,5 and 6 , the spreading rate of the jet as a function of the flight Mach number can be expressed empirically as,

$$
\beta=\arctan \left(\frac{0.5}{18.4 M_{\mathrm{f}}+5.3}\right)
$$

The spreading rate can then be used to define the shear layer width as a function of flight velocity,

$$
\delta_{\beta}= \begin{cases}\frac{0.93 x}{16 M_{\mathrm{f}}+4.6} & \text { if } x<X_{\mathrm{c}} \\ 0.5 D_{\mathrm{j}}\left(\frac{x}{16 M_{\mathrm{f}}+4.6}+1\right) & \text { if } x \geq X_{\mathrm{c}}\end{cases}
$$

The length of the jet's potential core and the jet spreading rate depend on the initial conditions present at the nozzle exit. Thus, in order to make Eq. 8 more general, the boundary layer region inside the core jet nozzle should be properly investigated. The main conclusion from Eq. 8 is that the variation of the shear layer width and, therefore, the characteristic length scale of an in-flight jet shear layer can be determined simply from the axial mean velocity profile along the jet centreline.

It is well-known that the mean velocity profiles of a jet under static ambient flow conditions are self-similar and scale with the half-velocity radius, $y_{0.5}$, and vorticity thickness, $\delta_{\omega}=U_{\mathrm{j}} /|\partial \bar{U} / \partial y|_{\max }$, parameters. Both the half-velocity radius and the maximum mean shear occur near the jet lipline at least two potential core lengths downstream of the nozzle exit and then eventually move towards the jet centreline. It can be seen that this self-similarity behaviour also holds for in-flight jets, see Fig. 8

The data presented in Fig. 8 collapses well in the inner part of the shear layer (i.e. $\left.\left(y-y_{0.5}\right)<0\right)$. As the flight velocity increases and the jet stretches, the edge of the shear layer moves towards the half-velocity-width location. The values $\left(y-y_{0.5}\right)>0$ at which data from in-flight jets do not collapse with the static jet curve are essentially points measured in the flight flow instead of the core jet. Similar results are seen when the shear layer width replaces the vorticity thickness as the local length.

\section{Turbulence Levels}

Both the single-point and the two-point statistics of the velocity fluctuation in the jet shear layer are typically used to inform jet mixing noise source models. The turbulence intensity in the streamwise direction is expected to decrease 


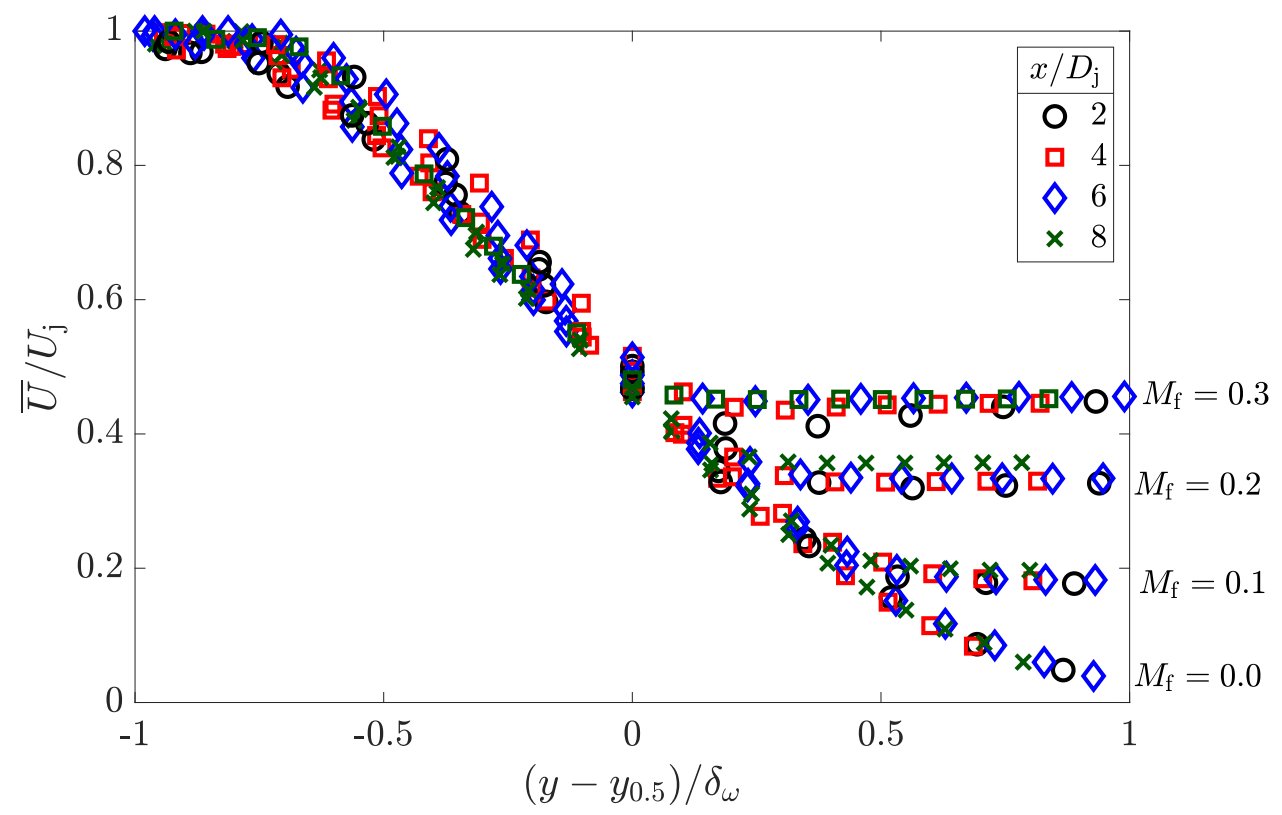

Fig. 8 Jet axial mean velocity profiles normalised by the shear layer jet half-velocity location and the vorticity thickness. Data taken at axial locations $x / D_{j}=2,4,6$, and 8 . Results for a $M_{j}=0.6$ static jet and three in-flight jets at $M_{f}=0.1,0.2,0.3$.

with increasing flight velocity at a given location because the mean shear between the jet and the ambient flow depends on the relative velocities of the two flows. The turbulence intensity discussed here is defined as the ratio between the root-mean-square of the velocity and the maximum mean velocity along the jet centreline. The turbulence intensity in the potential core of the flight jet is seen to be very low in comparison to the jet turbulence intensity (i.e. less than $1 \%$ ) [27].

The same trends were seen for both the axial and the radial unsteady velocity components. Thus, brief examples of the analysis based on the radial velocity fluctuation, $v$ are presented in Appendix A. In summary, it is possible to conclude that, within two jet potential core lengths, the turbulent kinetic energy reduces with increasing flight velocity. This turbulent kinetic energy reduction is in agreement with the decrease in sound pressure level seen in the far field of in-flight jets [7-9, 18]. The low turbulence levels in the flight jet potential core and the consistent trend in the behaviour of the jet turbulent kinetic energy with flight velocity are a strong indicative that open jet wind tunnels provide a good approximation to simulate forward flight effects on jet noise.

The potential core length and the shear layer width defined in Eq. 4 and Eq. 8 are used to normalise the radial distance of the profiles studied for both the static and in-flight jets. The turbulence levels were then normalised by the velocity difference between the jet and the ambient medium to account for the changes in the mean shear rate. The result is shown in Fig. 9 The root-mean-square of the velocity fluctuations is normalised by $U_{\mathrm{j}}-U_{\mathrm{f}}$ and the radial distance is normalised by the shear layer width defined in Eq. 8. A consistent data collapse is also seen at other axial locations 
when normalised relative to the jet's potential core lengths.

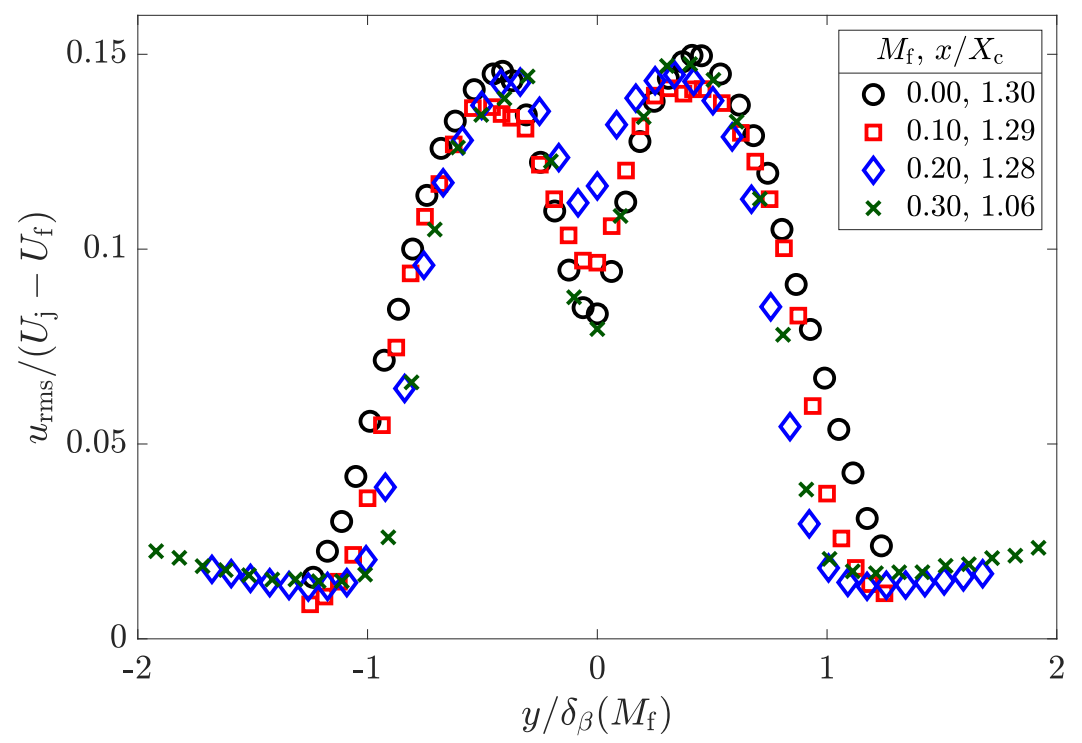

Fig. 9 Intensity of the axial velocity fluctuation normalised by the velocity difference $\left(U_{j}-U_{f}\right)$. The radial location is normalised by the shear layer width defined in Eq. 8 . Data for $M_{f}=0,0.1,0.2$, and 0.3 . The axial location of each profile relative to the potential core length, defined by Eq. 4 , is indicated in the legend

Although modelling the turbulence intensity profile is still a challenge for shear layer flows, results presented here show that the velocity characteristics of turbulence used for simple jet noise source models based on acoustic analogies can be simply modified to account for flight effects. Furthermore, the higher-order statistical moments of the in-flight jet configurations were seen to scale as per the low order statistics. Some examples of skewness and kurtosis distributions are presented in Appendix B.

\section{Power Spectral Density of the Unsteady Jet Velocity}

In this section, the power spectral densities (PSD) of the unsteady velocity field in the shear layer of in-flight jets are analysed and compared to those found for static jets. Although two-point measurements are necessary to obtain the frequency-dependent convection velocity and the flow characteristic length scales, the power spectral density is discussed here to determine the scaling of the frequency-dependent in-flight jet mixing noise source properties.

The shear-layer width parameter is used to collapse the peak frequency of the PSD at different axial locations along the lipline. As expected, the low frequency content of the power spectrum level increases with increasing axial location up to ten jet diameters [15, 28]. A dimensionless parameter accounting for this effect must be connected with a characteristic length-scale of the jet. Using the shear layer width defined in Eq. 8 , the following non-dimensional parameter is defined, 


$$
\mathrm{C}_{\delta}=\frac{\delta_{\beta}}{D_{\mathrm{j}}}
$$

A final normalisation to the PSD is applied to account for the effect of flight velocity. Here, the same velocity difference required to collapse the turbulence intensity data in Sec. [III.C is used. This factor is of the form,

$$
\mathrm{C}_{\mathrm{f}}=\frac{U_{\mathrm{j}}}{U_{\mathrm{j}}-U_{\mathrm{f}}}
$$

Results showing the velocity PSD of the static and in-flight jet configurations are shown in Fig. 10 Figure 10(a) displays data for a static jet at three axial locations and Fig. 10(b) shows the comparison between static and three in-flight jets. The data presented are at axial locations normalised by the jet potential core length, $x / X_{\mathfrak{c}}$. Note that the actual axial location shown varies in-flight as the potential core length increases with flight velocity (see discussion in Sec. III.B. Accordingly, the frequency is written as a Strouhal number based on the shear layer width, $\mathrm{St}_{\delta_{\beta}}$, and the PSD levels are normalised using the factors $C_{\delta}$ and $C_{\mathrm{f}}$.

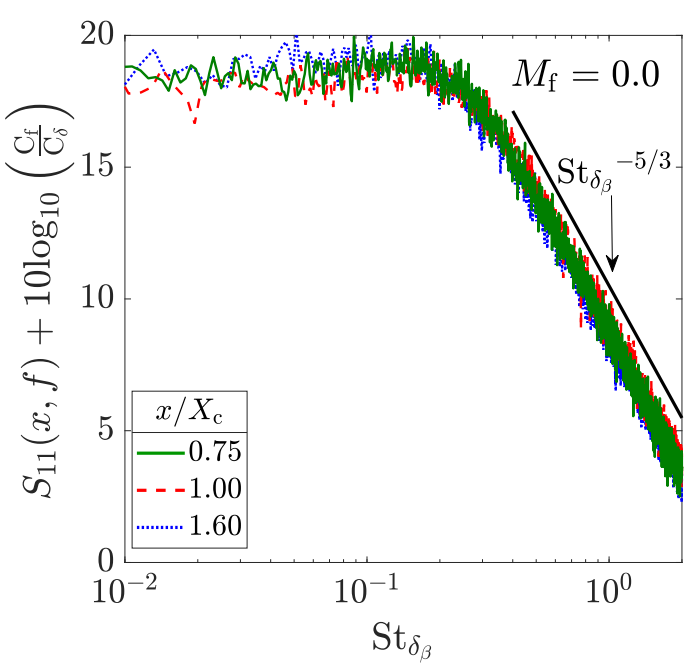

(a)

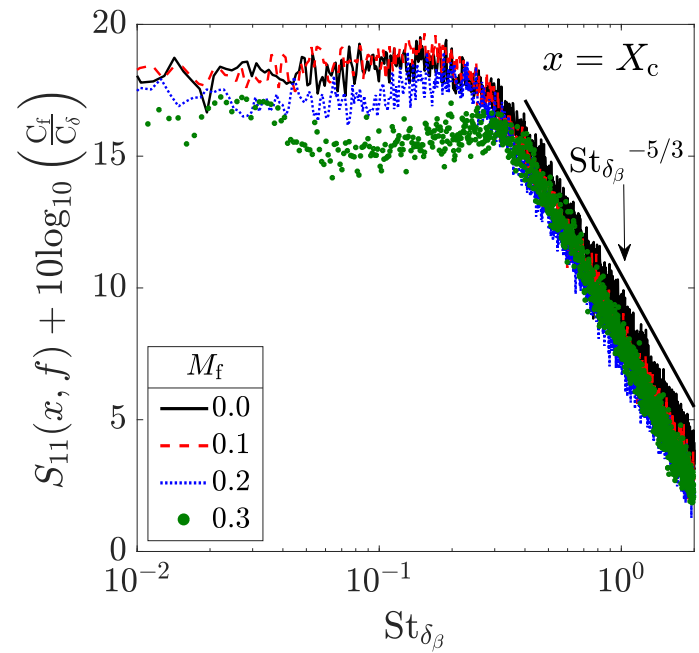

(b)

Fig. 10 Power spectral density normalised by shear layer width and flight velocity. Graph (a) displays three axial locations normalised by the jet potential core length, $M_{\mathrm{f}}=0.0$. Graph (b) shows data for static and three flight velocities measured at $x=X_{\mathrm{c}}$. Jet Mach number $M_{\mathrm{j}}=0.6$ and radial location $y / D_{\mathrm{j}}=0.5$

The scaling factors defined in Eq. 9 and in Eq. 10 provide a good collapse of the data for individual flight-stream velocities at all frequencies. The frequency cut-off between the flat low frequency energy-producing and the highfrequency inertial sub-range region is seen to be within $0.2 \leq \mathrm{St}_{\beta} \leq 0.3$. The PSD levels are seen to collapse particularly well for the static and lowest flight velocity case. As can be seen in Fig. 10(b), differences arise at $M_{\mathrm{f}}=0.2$ and $M_{\mathrm{f}}=0.3$, at which the low frequency PSD levels sit slightly lower in comparison to the other cases. Further investigation 
is necessary to explain this behaviour.

\section{E. Two-Point Statistics of the Jet Turbulent Field}

In this sub-section, the statistical joint moments of the in-flight jet are investigated. Autocorrelation coefficients are presented first followed by two-point functions in the time domain (i.e. cross-correlation) and then the frequency domain (i.e. coherence). The fixed, reference sensor used in this study was located on the lipline of the jet at the point of maximum turbulence intensity. The scaling laws presented here are not valid in highly-intermittent regions of the jet (i.e. the jet's potential core and near the edge of the jet shear layer). Appendix B presents a summary of jet locations at which results discussed herein are expected to be valid.

\section{Autocorrelation}

The autocorrelation coefficients are important as they are used to calculate the integral time-scales of the jet. The time-scale is obtained either from the area beneath the correlation curve or the time delay at which the correlation coefficient decays to 1/e, as suggested in the literature [15, 23, 24]. For points along the lipline where the turbulence kinetic energy is high, the correlation coefficients are seen to decay exponentially. Thus, the 1/e method provides the best accuracy for calculating the turbulence characteristic scales [15].

Fig. 11 depicts the autocorrelation coefficients for a static jet and the three in-flight jets $\left(M_{\mathrm{f}}=0.1,0.2\right.$, and 0.3$)$. Three axial locations on the lipline are shown normalised by the jet nozzle diameter, see Fig. 11(a) and by the jet potential core length, see Fig. 11(b) The coefficients shown in both graphs were obtained using the square of the velocity fluctuations.

The solid lines shown in Fig. 11] are the empirical trendlines established by collapsing the static jet data, see [15]. This shows that the flight Mach number-dependent shear layer width, defined in Eq. 8, can indeed be used to describe the development of the turbulent jet time-scales seen on the lipline for both static and in-flight ambient flow configurations. A consistent results was also found to exist for the second-order autocorrelation coefficients. As found for the static jet [15], both the second-order and the fourth-order coefficients are seen to behave as per homogeneous turbulence (i.e. $\left.R_{1111}(\tau)=R_{11}^{2}(\tau)\right)$

Noticeable deviations from the empirical model exist for points located close to the jet nozzle exit where the reference probe location was at $x / D_{\mathrm{j}}=2$ (green symbols in Fig. $11(\mathrm{a})$ ) and at the highest flight velocity, $M_{\mathrm{f}}=0.3$ (dot symbols in both subplots of Fig. 11). For the locations very close to the nozzle, the boundary layer that develops on the external surface of the jet pipe introduces an additional turbulence intensity in the flow field of the core jet. As discussed earlier, the external boundary layer effects are negligible at locations $x \geq 3 D_{\mathrm{j}}$. Another possible explanation for these discrepancies is the fact that the thin jet shear layer at $x / D_{\mathrm{j}}=2$ is affected by the signature of the Kelvin-Helmholtz instability seen in the potential core of both the core jet and the flight jet. The well-established 'hump' seen in the 


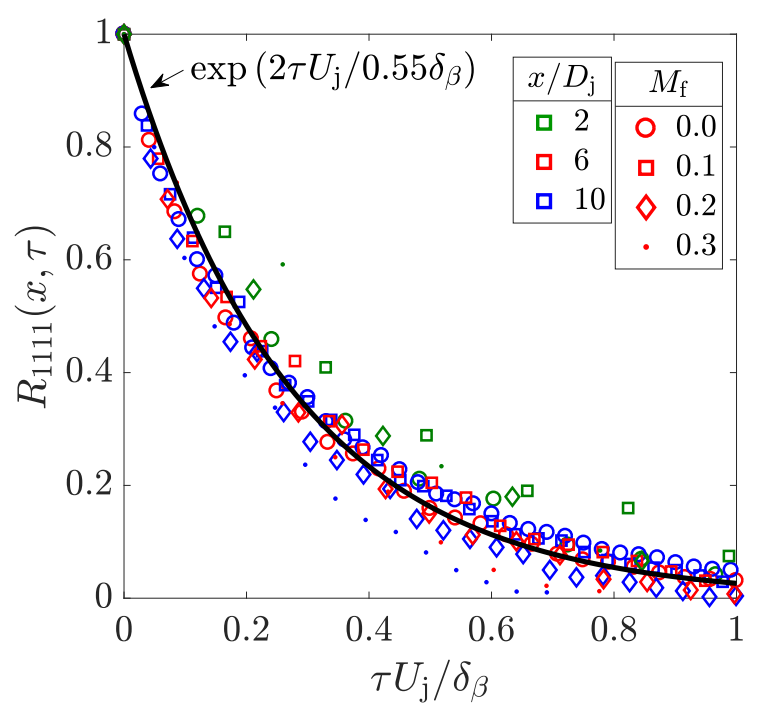

(a)

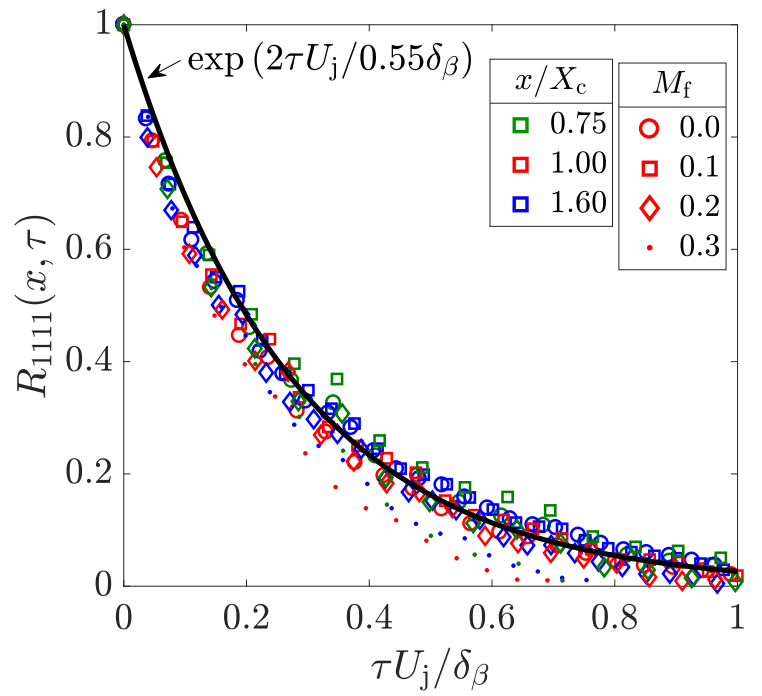

(b)

Fig. 11 Autocorrelation coefficients measured along the jet lipline. Several axial locations are shown normalised by: a) the jet diameter, and b) the jet potential core length. Axial locations are differentiated by the different marker colours. Results for four flight Mach numbers indicated by the different symbols

potential core of jets appears as a oscillatory function in correlation coefficients. As the flight velocity increases, the flight jet potential core hump frequency increases and the jet shear layer becomes thinner, both effects contributing to a slower decay of the correlation coefficients.

In locations further downstream, the working hypothesis for the differences seen of in-flight $M_{\mathrm{f}}=0.3$ jet is the increase in the temperature due to the high power setting in the fan generating the flight flow. As the flight temperature increases during measurements, the nominal flight velocity varies. The shear layer width at a given axial location differs from that calculated using Eq. 8, as an inaccurate flight Mach number is used. Additionally, the total temperature of the jet and flight flows are substantially different (e.g. around $11^{\circ} \mathrm{C}$ after running both rigs for ten minutes). The farthest downstream the jet nozzle exit, the more complex is the mixing between the jet and flight flows. Thus, the temperature correction applied to the hot-wire signal is likely to be unsuccessful in that region. To avoid the issues associated with the flight temperature increase, only data up to $M_{\mathrm{f}}=0.2$ will be used in the subsequent analysis.

Despite these minor departures from the model for static jets, the time-scales for the in-flight cases collapse well. It is also interesting to note that the results shown in Fig. 11 using Eq. 8 are in good agreement even though the data presented in this paper and that of the literature for static jets [15] were obtained from different jet nozzles. Not only the diameters of both jet nozzles are different $(38.1 \mathrm{~mm}$ for static data and $40 \mathrm{~mm}$ for in-flight measurements), but also the internal contours: the $38.1 \mathrm{~mm}$ nozzle has a high convergence angle $\left(14^{\circ}\right)$, whilst the $40 \mathrm{~mm}$ nozzle used in the in-flight experiments has a convergence half-angle of only $3^{\circ}$. The boundary layer at the exit of the $40 \mathrm{~mm}$ nozzle is around three times thicker than the boundary layer in the $38.1 \mathrm{~mm}$ nozzle [18]. Nonetheless, in the quasi-Gaussian region (see 
Appendix B), the characteristic time and length scales are similar.

\section{Space-Time Cross-Correlation and Eddy Convection Velocity}

The model presented by Proença et al. [15] for the axial cross-correlation coefficients of a static jet are also seen to be valid for in-flight jets when the correct scaling parameters are used. Figure 12(a) shows an example of the axial cross-correlation coefficients for the static and in-flight jet cases investigated. Five axial separation distances are shown and the jet stretching effect is correctly captured by normalising with the local shear layer width. Although the curves do not completely collapse, the location of the peaks is well defined for all data. Thus, the Moving frame autocorrelation envelope can be adequately described by a single exponential function. Figure 12(b) shows the autocorrelation moving frame at the different locations and two flight speeds analysed.

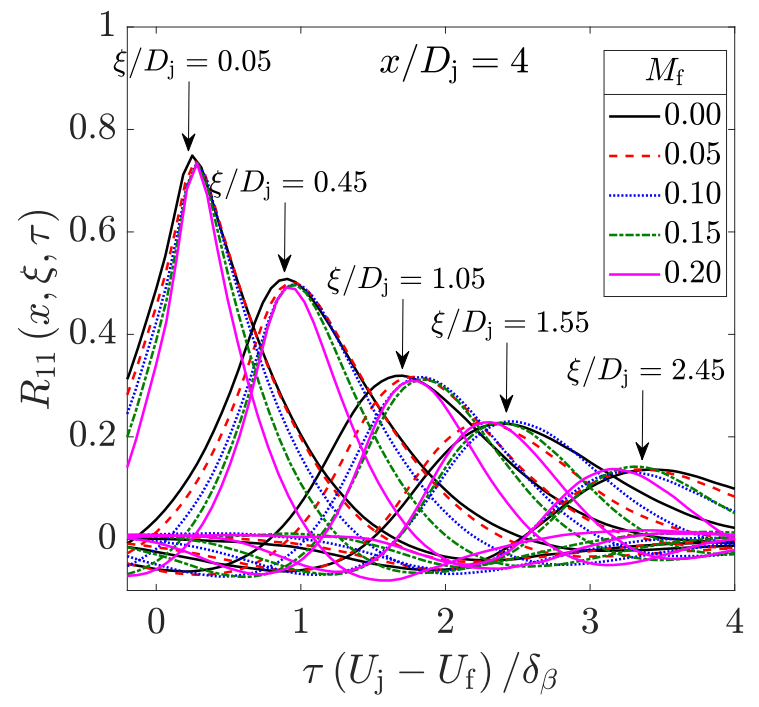

(a)

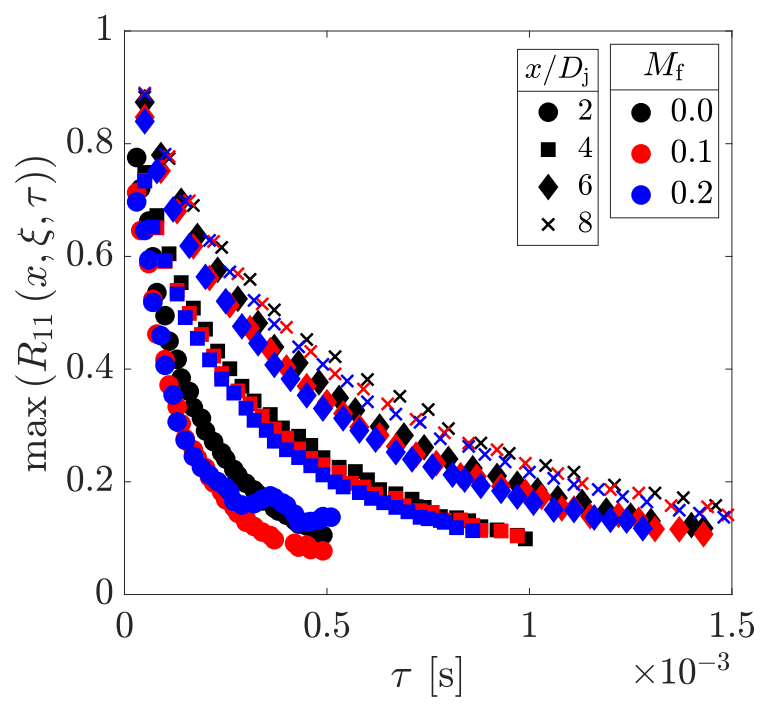

(b)

Fig. 12 Second-order space-time cross-correlation coefficients of the streamwise component of the jet velocity: (a) correlation curves for five separation distances and five flight velocities, reference sensor located at $\mathrm{x} / \mathrm{Dj}=4$ and $y / D_{j}=0.5$; (b) peak cross-correlation coefficients for four reference sensor locations and three flight velocities

It is concluded from Fig. 12 that the moving frame of reference of static and in-flight jets scale with the velocity difference and shear layer width. An example of using these parameters to collapse static and in-flight jet space-time cross-correlation coefficients is shown in Fig. 13(a), Data corresponding to the same four reference sensor locations and three flight velocities are displayed.

In Fig. 12(a) the velocity difference and shear layer width are seen to collapse the data peaks well (i.e. the moving frame of reference). The shape of the bell curves, however, are not well collapsed. Thus, when the space correlation coefficients are considered (i.e. fixed frame of reference, $R(\tau=0)$ ) the effect of the velocity difference should be 


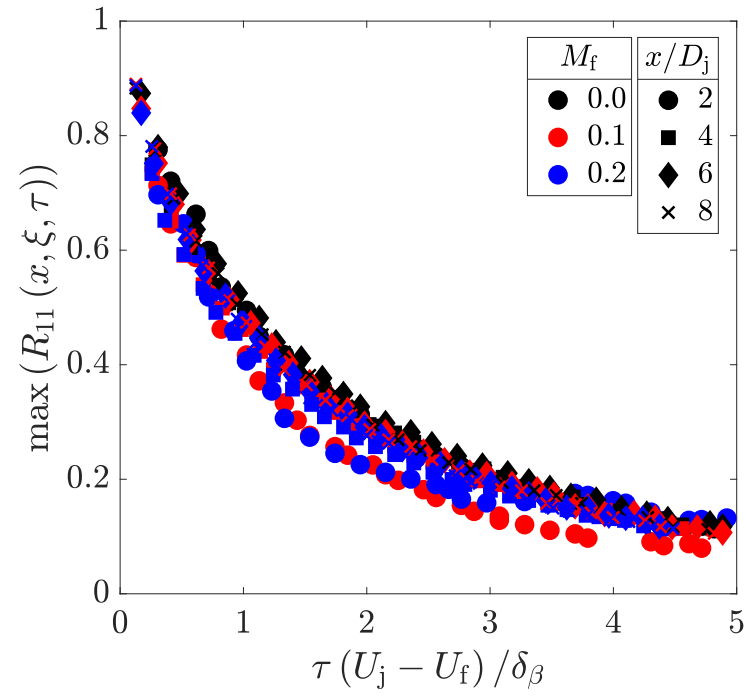

(a)

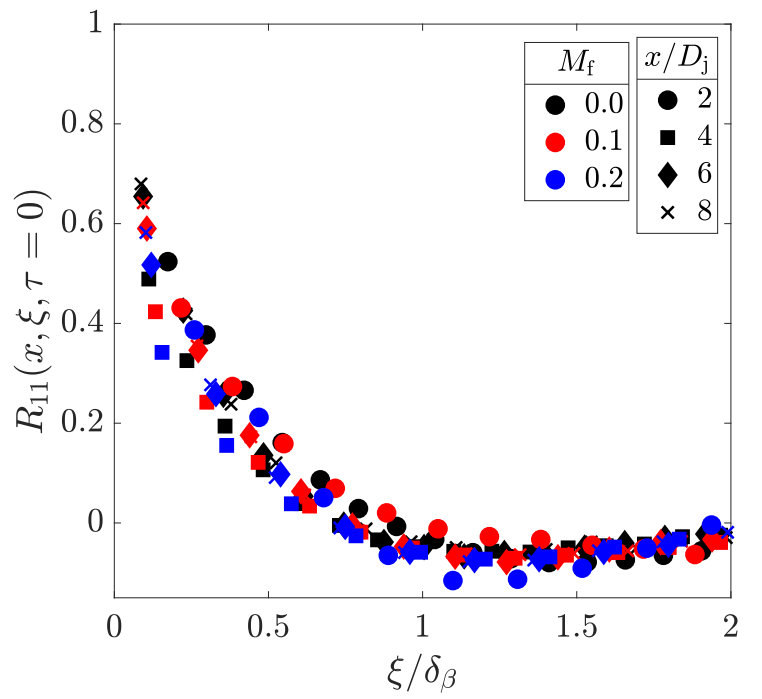

(b)

Fig. 13 Second-order cross-correlation coefficients: (a) space-time coefficients in the moving frame of reference. (b) space coefficients in the fixed frame of reference. Both graphs depict data at four axial locations and at three flight velocities

negligible. To demonstrate this, the non-normalised space correlation coefficients were evaluated and the separation distance was then normalised by the shear layer width at the reference sensor position. Space correlation coefficients for one static and two in-flight jets are shown in Fig. 13(b). Four reference sensor axial locations are displayed. The characteristic length scale of static and in-flight jets is estimated to be $0.25 \delta_{\beta}$, where $\delta_{\beta}$ is as defined in Eq. 8

Finally, the eddy convection velocities of the various configurations were calculated using the separation distance and time delay corresponding to the peaks of the cross-correlation coefficients in Fig. 12(a). Figure 14 displays the locations of the coefficient peaks as a function of separation distance and time. Three best-fit convection velocity lines, equal to $0.55 U_{\mathrm{j}}, 0.6 U_{\mathrm{j}}$ and $0.65 U_{\mathrm{j}}$, are shown.

It can be seen that the convection velocity increases consistently with flight Mach number. This can be explained by the decrease in mean shear between the jet and the ambient medium as flight velocity is increased. An empirical equation for the variation of the eddy convection velocity along the lipline with jet-flight velocity ratio is defined by,

$$
\frac{U_{\mathrm{c}}}{U_{\mathrm{j}}}=0.3 \frac{U_{\mathrm{f}}}{U_{\mathrm{j}}}+0.55
$$

\section{Coherence and Frequency Dependent Length-Scales}

The cross-power spectral density is used here to evaluate the coherence function and frequency-dependent lengthscales within the jet flow. Figure $15(\mathrm{a})$ shows an example of the magnitude of the coherence measured at four separation 


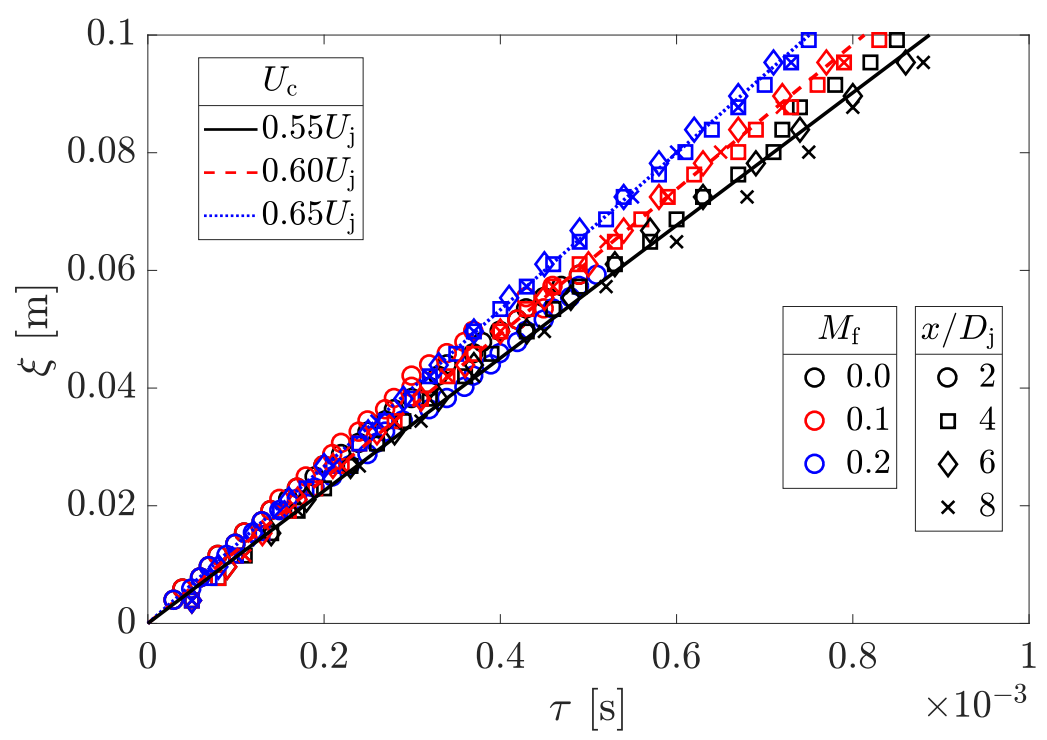

Fig. 14 Calculated eddy convection velocity for one static and two in-flight jets at four axial locations based on the peaks of the cross-correlation coefficients

distances and at three flight velocities. In this example, the reference sensor was located at $x / D_{\mathrm{j}}=4$. The low frequency energy is seen to be independent of frequency up until a point where the coherence decays with the inverse of frequency. At a given frequency, a frequency-dependent length-scale can be defined using the decay of the coherence with increasing separation distance [24]. For static jets, as the axial separation increases, the coherence is seen to decay exponentially [15, 23, 24]. This behaviour is also observed for the in-flight jet cases studied here. Thus, the frequency-dependent length-scale, $\Lambda$, can be obtained using the 1/e method. Figure $15(\mathrm{~b})$ displays the variation of $\Lambda$ with frequency at several axial locations and flight velocities. The same parameters used to collapse the space-time cross-correlation coefficients are also used here resulting in a good collapse.

The main discrepancy to the data collapse in Fig. 15 corresponds to when the reference sensor was located at $x / D_{\mathrm{j}}=2$. The explanation for this behaviour is likely to be related to the nozzle external boundary layer. At $x / D_{\mathrm{j}}=2$, only one flight velocity, $M_{\mathrm{f}}=0.2$ is shown to improve clarity. The effects of the nozzle external boundary layer are seen at frequencies $\mathrm{St}_{\beta}>0.1$. The frequency-dependent length-scales obtained for in-flight jets are otherwise seen to collapse as per the static case. This further evidences the fact that the velocity difference and the shear layer width parameters both can be used to predict single-point and two-point statistics of an in-flight jet up to $M_{\mathrm{f}}=0.2$.

\section{Conclusion}

In this paper, an experimental investigation into the turbulence statistics of an in-flight jet has been presented. The experiments were performed using the new Doak Flight Jet Rig at the ISVR. A Mach 0.6 single-stream core jet was surrounded by a flight-stream flow up to Mach 0.3. Single-point hot-wire measurements have been analysed and 


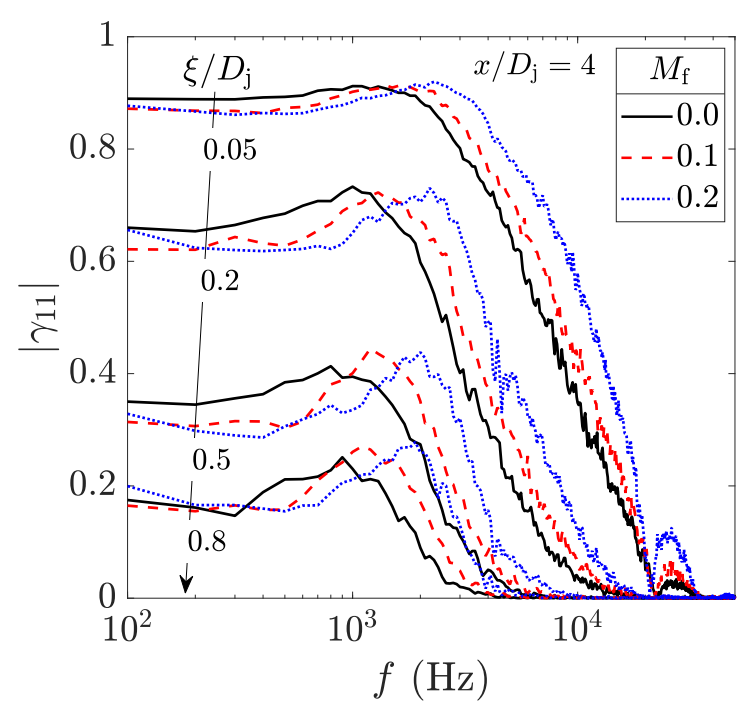

(a)

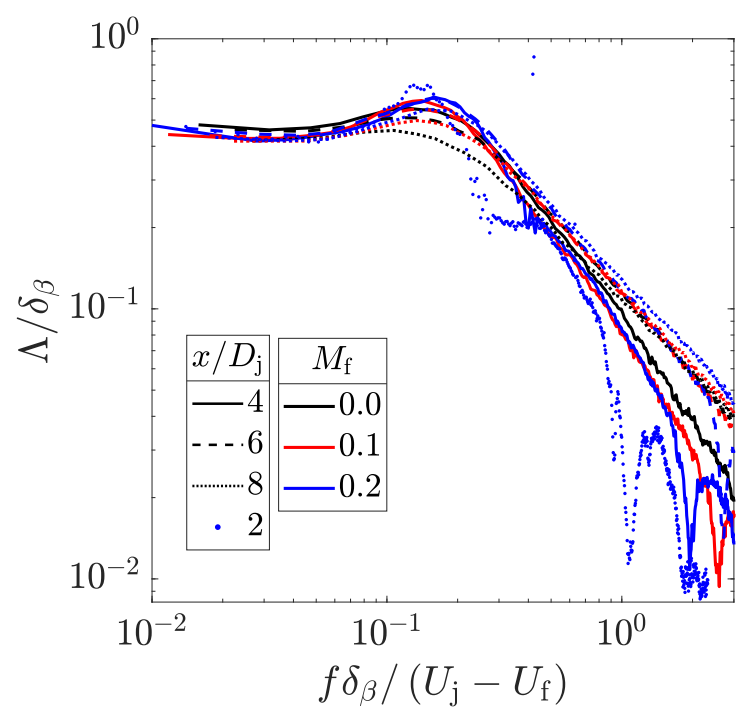

(b)

Fig. 15 Two-point statistics in the frequency domain: (a) magnitude of the coherence function, reference sensor located at $x / D_{j}=4$, for three flight Mach numbers using four axial separation distances. (b) Frequencydependent length-scales obtained from the decay of the magnitude of the coherence function

discussed, and an empirical model for the stretching of an in-flight jet has been developed. Additionally, self-similar scaling parameters have been found for both the low-order statistical moments (i.e. autocorrelation, power spectral density) and the joint moments, including the correlation and coherence functions. The data suggest that the same static jet assumptions can be used to model in-flight jets.

Thus, the characteristic length-scale produced by an in-flight jet remains proportional to the jet shear layer width. Since the mean axial velocity remains self-similar, the length-scales can be found from the degree with which the jet stretches with increasing flight velocity. The characteristic time-scale, however, is seen to vary proportionally with the ratio of the shear layer width and the velocity difference, $U_{\mathrm{j}}-U_{\mathrm{f}}$. With these parameters, recent empirical models for the single-point and two-point statistics of static jets [15] can now be used to account for forward flight effects.

Furthermore, the eddy convection velocity is seen to increase proportionally with increasing flight velocity. This is assumed to be due to the decrease in mean shear in the outer core jet shear layer. As expected, from the statistical analysis, the eddy convection velocity was seen to behave proportionally to the velocity difference, $U_{\mathrm{j}}-U_{\mathrm{f}}$. Therefore, the two-point statistics has the same self-similar parameters as the single-point statistics.

The single-point and two-point statistical models presented in this paper can be used for the prediction of in-flight jet mixing noise. They will also play an important role in inferring in-flight jet-surface interaction noise models. 


\section{Appendix}

\section{A. Radial Component of the Jet Velocity Field}

Single-point measurements were also performed using cross-wire probes to study the radial component of the velocity field. Overall, similar trends were seen for the longitudinal and transverse velocity components. In this section, key results using the radial velocity fluctuation data are briefly discussed.

The mean velocity of the $v$ and $w$ components is known to be low compared to the axial mean velocity, $u$. As expected, the mean radial velocity peaks on the lipline, near the nozzle exit and it is $5 \%$ of the jet exit velocity. As flight velocity increases, the mean radial velocity and turbulence intensity development behaves as discussed in Section III.B.

It is also well-established that, in comparison to the axial velocity, the transverse turbulence intensities are about $3 \%$ lower than the axial turbulence intensity [23]. Nevertheless, the scaling of the turbulence intensity profiles for the in-flight jets using the shear layer width and velocity difference parameters still appears to be valid. Figure 16 displays turbulence intensity profiles of both the axial and radial components of the velocity field for three flight velocities. Note that the axial jet locations relative to the jet potential core do not match. Thus, the data do not collapse close to the jet centreline.

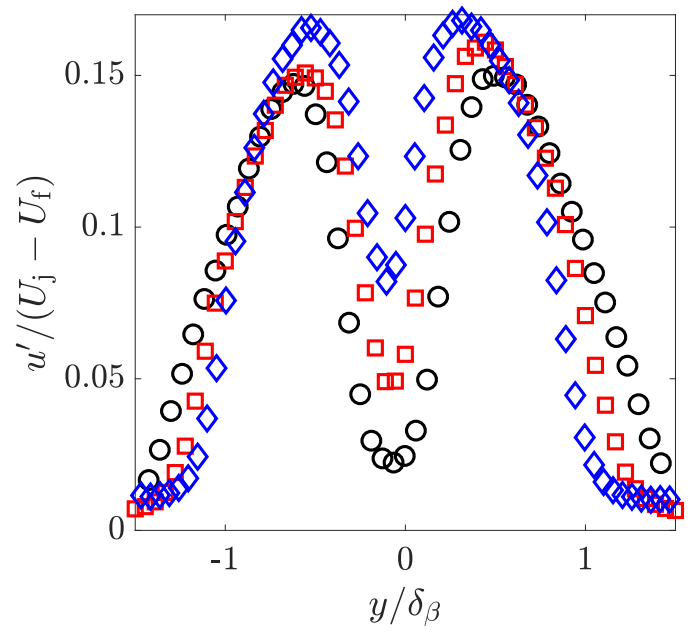

(a)

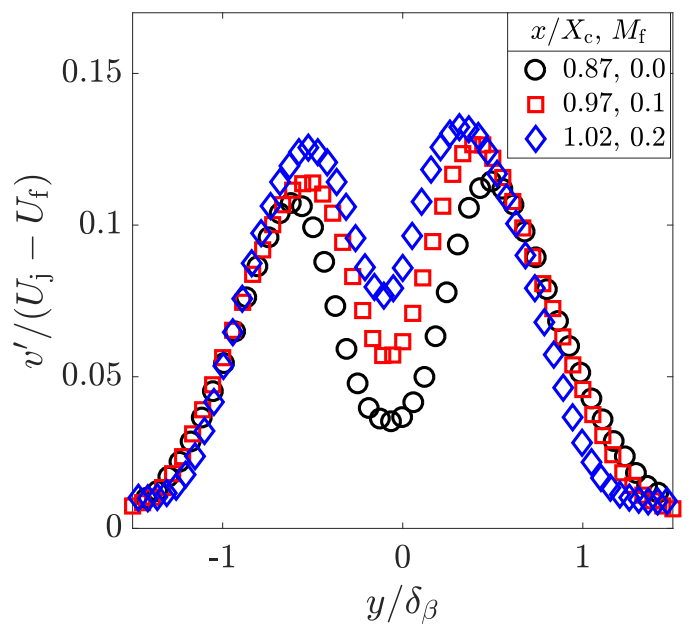

(b)

Fig. 16 Scaling of the root-mean-square of the velocity fluctuation: (a) axial component of the velocity; (b) radial component of the velocity. $M_{j}=0.6$

As expected, the power spectral density of both the $u$ and $v$ velocity components display similar behaviour.

\section{B. Higher-order Moments}

Simplifying assumptions about the flow can be used to infer the single-point and two-point statistical properties at different locations of the jet flow. Here it is assumed that: 1) the region of maximum turbulence intensity remains close 
to the lipline up to two jet's potential core and 2) the velocity fluctuation has a quasi-normal distribution in this region. Higher-order statistical moments are used to define the validity of the assumptions (1) and (2). Figure 17] shows the distributions of the skewness and kurtosis of the static jet and $M_{\mathrm{f}}=0.2$ in-flight jet. The dashed lines illustrate the regions where the distribution is symmetric (i.e. when the skewness is zero) and normal distribution (i.e. when the kurtosis is three).
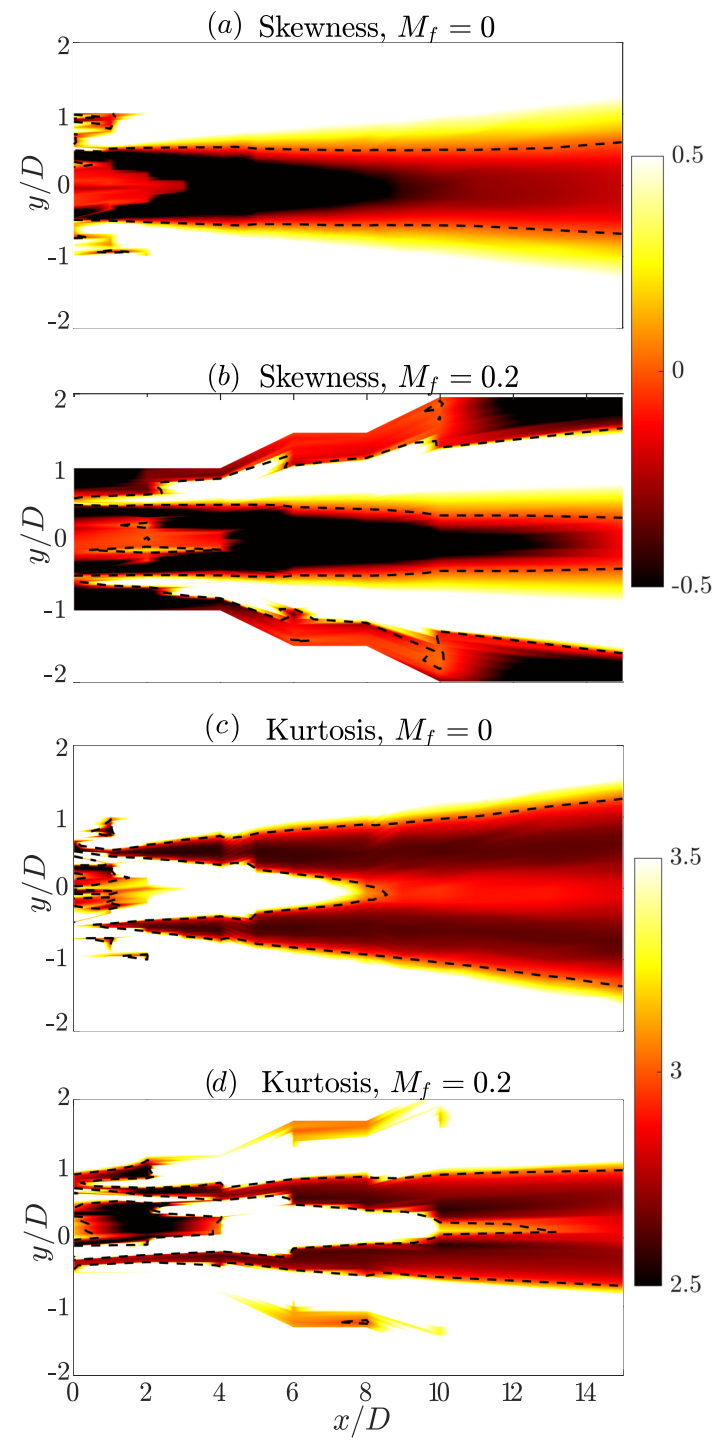

Fig. 17 Higher-order statistics of the static (a,c) and in-flight jets (b,d). Skewness (a,b) and kurtosis (c,d) are plotted

The trends seen in Fig. 17 could have been inferred from the low order mappings. The potential core region and locations near the edge of the shear layer are highly intermittent and deviate substantially from a normal distribution. The flight-speed increases the potential core and stretches the jet, therefore, the region in which a quasi-Gaussian distribution applies becomes narrow with increasing flight-stream velocity. Thus, the assumptions used in the paper 
of homogeneous and frozen turbulence are valid for points along the lipline. In these locations, the data shows that second-order and fourth-order two-point coefficients are related as $R_{1111}=R_{11}^{2}$ and $\gamma_{1111}=\gamma_{11}^{2}$, and the eddy convection velocity is similar to the jet local mean velocity.

Integral time and length scales of in-flight jets were shown to be simply calculated from the physical dimensions of the jet as per the static jet case. Although not much evidence can be provided to the behaviour of the transverse length scales with changes in the surrounding medium velocity, the hypothesis that the stretching of the shear layer width also accounts for the changes in the radial and azimuthal length scales is reasonable.

\section{Acknowledgments}

A. R. Proença would like to acknowledge financial support from the CAPES Foundation within the Brazilian Ministry of Education (Grant BEX-9333-13-4).

\section{References}

[1] Bailly, C., Bogey, C., and Castelain, T., Subsonic and Supersonic Jet Mixing Noise - in Measurement, Simulation and Control of Subsonic and Supersonic Jet Noise, von Karman Institute for Fluid Dynamics, 2016.

[2] Camussi, R., Noise Sources in Turbulent Shear Flows: Fundamentals and Applications, Springer-Verlag Wilen, CISM, Udine, 2013.

[3] Southern, I., "Exhaust noise in flight - The role of acoustic installation effects," 6th Aeroacoustics Conference, American Institute of Aeronautics and Astronautics, 1980. doi:10.2514/6.1980-1045, URL https://arc.aiaa.org/doi/abs/10. $2514 / 6.1980-1045$

[4] Reddy, N., and Tanna, H., "Installation effects in jet noise in flight," 6th Aeroacoustics Conference, American Institute of Aeronautics and Astronautics, 1980. doi:10.2514/6.1980-1044, URLhttps://arc.aiaa.org/doi/abs/10.2514/6.19801044

[5] Miller, W., "Flight effects for jet-airframe interaction noise," 8th Aeroacoustics Conference, American Institute of Aeronautics and Astronautics, 1983. doi:10.2514/6.1983-784, URL/https://arc . aiaa.org/doi/abs/10.2514/6.1983-784.

[6] Mead, C., and Strange, P., "Under-wing installation effects on jet noise at sideline," 4th AIAA/CEAS Aeroacoustics Conference, American Institute of Aeronautics and Astronautics, 1998. doi:10.2514/6.1998-2207, URL https://arc.aiaa.org/doi/ abs/10.2514/6.1998-2207

[7] Cocking, B., and Bryce, W., "Subsonic jet noise in flight based on some recent wind-tunnel tests," 2nd Aeroacoustics Conference, American Institute of Aeronautics and Astronautics, 1975. doi:10.2514/6.1975-462, URL https://arc.aiaa.org/doi/ abs/10.2514/6.1975-462 
[8] Ahuja, K., Tanna, H., and Tester, B., "An experimental study of transmission, reflection and scattering of sound in a free jet flight simulation facility and comparison with theory," Journal of Sound and Vibration, Vol. 75, No. 1, 1981, pp. 51 - 85. doi:https://doi.org/10.1016/0022-460X(81)90235-2, URL http://wwW.sciencedirect.com/science/article/ pii/0022460X81902352.

[9] Viswanathan, K., "Progress in prediction of jet noise and quantification of aircraft/engine noise components," International Journal of Aeroacoustics, Vol. 17, No. 4-5, 2018, pp. 339-379. doi:10.1177/1475472X18778279, URL https://doi .org/ $10.1177 / 1475472 \times 18778279$

[10] Drevet, P., Duponchel, J., and Jacques, J., "The effect of flight on jet noise as observed on the Bertin Aérotrain,” Journal of Sound and Vibration, Vol. 54, No. 2, 1977, pp. 173 - 201. doi:https://doi.org/10.1016/0022-460X(77)90022-0, URL http://www.sciencedirect.com/science/article/pii/0022460X77900220

[11] Morris, P. J., "Turbulence Measurements in Subsonic and Supersonic Axisymmetric Jets in a Parallel Stream,” AIAA Journal, Vol. 14, No. 10, 1976, pp. 1468-1475. doi:10.2514/3.61485, URLhttps://doi.org/10.2514/3.61485

[12] Tanna, H., and Morris, P., "In-flight simulation experiments on turbulent jet mixing noise," Journal of Sound and Vibration, Vol. 53, No. 3, 1977, pp. 389 - 405. doi:https://doi.org/10.1016/0022-460X(77)90422-9, URL http://www. sciencedirect. com/science/article/pii/0022460X77904229

[13] Plumblee, H. E., Jr., "Effects of forward velocity on turbulent jet mixing noise," Tech. rep., Jul. 1976. URL https: //ui.adsabs.harvard.edu/abs/1976lock.rept.....P

[14] Larson, R. S., MeColgan, C. J., and Packman, A. B., "Jet Noise Source Modification Due to Forward Flight," AIAA Journal, Vol. 16, No. 3, 1978, pp. 225-232. doi:10.2514/3.60881, URL https://doi.org/10.2514/3.60881.

[15] Proença, A., Lawrence, J., and Self, R., "Measurements of the single-point and joint turbulence statistics of high subsonic jets using hot-wire anemometry," Experiments in Fluids, Vol. 60, No. 4, 2019, p. 63. doi:10.1007/s00348-019-2716-3, URL https://doi.org/10.1007/s00348-019-2716-3

[16] Bryce, W., "The prediction of static-to-flight changes in jet noise," 9th Aeroacoustics Conference, American Institute of Aeronautics and Astronautics, 1981. doi:10.2514/6.1984-2358, URLhttps://arc . aiaa.org/doi/abs/10.2514/6.19842358

[17] Lawrence, J., and Self, R., "Installed jet-flap impingement tonal noise," 21st AIAA/CEAS Aeroacoustics Conference, 2015. doi:10.2514/6.2015-3118, URL/https://arc.aiaa.org/doi/abs/10.2514/6.2015-3118

[18] Proença, A. R., "Aeroacoustics of isolated and installed jets under static and in-flight conditions," Ph.D. thesis, University of Southampton, March 2018. URL https://eprints.soton.ac.uk/426880/

[19] Jørgensen, F. E., "How to Measure Turbulence with Hot-Wire Anemometers - a Practical Guide," Technical Report, Dantec Dynamics, Skovlunde, Denmark, 2002. 
[20] Wang, Z.-N., Proença, A., Lawrence, J., Tucker, P., and Self, R., "LES Prediction of an Installed Jet Flow and Noise with Experimental Validation," submitted to the AIAA Journal, 2020.

[21] Hussein, H. J., Capp, S. P., and George, W. K., "Velocity measurements in a high-Reynolds-number, momentum-conserving, axisymmetric, turbulent jet," Journal of Fluid Mechanics, Vol. 258, 1994, p. 31-75. doi:10.1017/S002211209400323X.

[22] Rajaratnam, N. (ed.), Chapter 1 The Plane Turbulent Free Jet, Developments in Water Science, Vol. 5, Elsevier, 1976. doi:https://doi.org/10.1016/S0167-5648(08)70901-8, URL http://www.sciencedirect.com/science/article/pii/ S0167564808709018.

[23] Harper-Bourne, M., "Jet noise turbulence measurements," 9th AIAA/CEAS Aeroacoustics Conference and Exhibit, 2003. doi:https://doi.org/10.2514/6.2003-3214, URL https://arc.aiaa.org/doi/10.2514/6.2003-3214

[24] Morris, P. J., and Zaman, K., "Velocity measurements in jets with application to noise source modeling," Journal of Sound and Vibration, Vol. 329, No. 4, 2010, pp. 394 - 414. doi:http://dx.doi.org/10.1016/j.jsv.2009.09.024, URL https: //wWW.sciencedirect.com/science/article/pii/S0022460X09007482?via\%3Dihub

[25] Witze, P. O., "Centerline Velocity Decay of Compressible Free Jets,” AIAA Journal, Vol. 12, 1974, pp. 417-418. doi: https://doi.org/10.2514/3.49262, URL https://arc .aiaa.org/doi/10.2514/3.49262.

[26] Forstall, W., and Shapiro, A. H., "Momentum and Mass Transfer in Coaxial Gas Jets," Journal of Applied Mechanics, Vol. 17, No. 4, 1950, pp. 399-408.

[27] Proença, A., Lawrence, J., and Self, R., "Experimental Investigation into the Turbulence Flow Field of In-Flight Jets,” 25th AIAA/CEAS Aeroacoustics Conference, 2019. doi:10.2514/6.2019-2545, URL https://arc . aiaa.org/doi/abs/10.2514/ $6.2019-2545$

[28] Laurence, J. C., "Intensity, Scale, and Spectra of Turbulence in Mixing Region of Free Subsonic Jet,” NACA TR $1292,1956$. URL https://ntrs.nasa.gov/archive/nasa/casi.ntrs.nasa.gov/19930092288.pdf 